\title{
From Coxeter higher-spin theories to strings and tensor models
}

\author{
M.A. Vasiliev \\ I.E. Tamm Department of Theoretical Physics, Lebedev Physical Institute, \\ Leninsky prospect 53, 119991, Moscow, Russia \\ E-mail: vasiliev@lpi.ru
}

\begin{abstract}
A new class of higher-spin gauge theories associated with various Coxeter

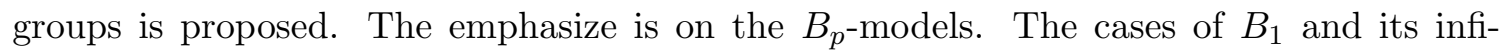
nite graded-symmetric product sym $\left(\times B_{1}\right)^{\infty}$ correspond to the usual higher-spin theory and its multi-particle extension, respectively. The multi-particle $B_{2}$-higher-spin theory is conjectured to be associated with String Theory. $B_{p}$-higher-spin models with $p>2$ are anticipated to be dual to the rank- $p$ boundary tensor sigma-models. $B_{p}$ higher-spin models with $p \geq 2$ possess two coupling constants responsible for higher-spin interactions in AdS background and stringy/tensor effects, respectively. The brane-like idempotent extension of the Coxeter higher-spin theory is proposed allowing to unify in the same model the fields supported by space-times of different dimensions. Consistency of the holographic interpretation of the boundary matrix-like model in the $B_{2}$-higher-spin model is shown to demand $N \geq 4$ SUSY, suggesting duality with the $N=4$ SYM upon spontaneous breaking of higher-spin symmetries. The proposed models are shown to admit unitary truncations.
\end{abstract}

KeYwords: AdS-CFT Correspondence, Higher Spin Gravity, Higher Spin Symmetry

ARXIV EPRINT: 1804.06520

To my father 


\section{Contents}

1 Introduction 2

2 Standard higher-spin equations $\quad 6$

3 Deformed oscillator algebras $\quad 7$

$\begin{array}{lll}3.1 & \text { Single oscillator } & 7\end{array}$

3.2 Many oscillators 8

3.3 Coxeter groups and Cherednik algebras 9

4 Higher-spin modules and framed oscillator algebras 11

5 Framed Cherednik algebras 13

$5.1 A_{N}$

$\begin{array}{lll}5.2 & \text { General Coxeter group } & 14\end{array}$

6 Coxeter higher-spin equations $\quad 14$

6.1 General scheme 14

$\begin{array}{lll}6.2 & \text { Spinor models } & 16\end{array}$

$\begin{array}{lll}\text { 6.2.1 } 3 d \text { Coxeter higher-spin models } & 17\end{array}$

$\begin{array}{ll}6.2 .24 d \text { Coxeter higher-spin models } & 18\end{array}$

$\begin{array}{lll}6.3 & \text { Vector Coxeter higher-spin models in any dimension } & 19\end{array}$

$\begin{array}{lll}7 & \text { Higher forms and invariants } & 21\end{array}$

8 Color extensions $\quad 23$

9 Idempotent extension $\quad 24$

9.1 General scheme 24

9.2 Realization 25

9.2.1 Vector-like models 25

$\begin{array}{ll}9.2 .2 N=4 \text { SUSY } & 27\end{array}$

10 Unitarity 28

11 Field frames of multi-particle theories $\quad 30$

$\begin{array}{ll}11.1 & \text { Definition and field equations } \\ \end{array}$

11.2 Frame generating functions 32

$\begin{array}{ll}11.3 \text { Unity idempotent map } & 35\end{array}$

12 Interpretation $\quad 36$

12.1 Strings and tensor models 36

$\begin{array}{ll}12.2 \text { Space-time metamorphoses } & 39\end{array}$

13 Conclusion $\quad 41$ 


\section{Introduction}

Higher-spin (HS) gauge theories, that describe interactions of massless fields of all spins, provide an interesting arena for testing principles of fundamental physics and, in particular, the $A d S / C F T$ correspondence conjecture [1-3]. First example of fully nonlinear HS theory was given for the $4 d$ case in [4], while its modern formulation was worked out in [5] (see [6] for a review). A specific property of HS gauge theories is that consistent interactions of propagating massless fields demand a curved background providing a length scale in HS interactions that contain higher derivatives. $(A) d S$ is the most symmetric curved background compatible with HS interactions [7]. The $A d S_{4} \mathrm{HS}$ model is the simplest nontrivial one in the sense that $d=4$ is the lowest dimension where HS massless fields propagate.

There were several long-standing problems in HS theories. One was the issue of locality raised originally in $[8,9]$ where it was shown that current interactions in HS theories can be removed by a seemingly local field redefinition containing infinite power series in higher derivatives with the coefficients containing inverse powers of the cosmological constant. This issue has been further analyzed in a number of papers [10]-[16]. The conclusion of $[14,15]$ was that unfolded HS equations lead to correct local interactions in the lowest order. These results are now extended to higher orders due to the development of the appropriate homotopy technics in $[17,18]$ where it is explained in particular how to identify the minimally nonlocal frame of [15] and to decrease the level of nonlocality in HS equations of [5] in higher orders.

Once the problem of locality in HS gauge theory has been sorted out, the fundamental remaining question is how to construct more general HS models that could be related to String Theory [19]. This is the problem addressed in this paper where we propose a new class of HS-like gauge theories that contain richer spectra of fields than the standard models of $[5,8]$. The proposed models are argued to be rich enough to lead to String Theory-like models with massive HS fields via spontaneous breakdown of HS symmetries. A concrete proposal for the realization of this idea briefly discussed in the end of this paper can shed light on the origin of the most symmetric phase of String Theory discussed long ago in [20,21]. (For related discussion see also [22]-[25].) Although this conjecture was supported by the analysis of high-energy limit of string amplitudes [21] and passed some nontrivial tests [26]-[29], no satisfactory understanding of this relation beyond the free field sector of the tensionless limit of String Theory [30-32] was available. Note however that an interesting idea of the singleton string whose spectrum is represented by multiple tensor products of singletons was put forward in [33, 34].

The idea of our construction came from the recent development of the AdS/CFT holography [1-3]. Indeed, the fact that HS theories are most naturally formulated in the AdS background was conjectured to play a role in the context of $A d S / C F T$ correspondence [35][39]. This expectation conforms to the fundamental result of Flato and Fronsdal [40] on the relation between tensor products of $3 d$ conformal fields (singletons) and infinite towers of $4 d$ massless fields that appear in HS theories.

In the important work of Klebanov and Polyakov [41] it was argued that the HS gauge theory of [5] should be dual to the $3 d O(N)$ sigma-model in the $N \rightarrow \infty$ limit. The 
Klebanov-Polyakov conjecture was checked by Giombi and Yin in [42, 43] where it was shown in particular how the bulk computation in HS gauge theory reproduces at least some of conformal correlators in the free $3 d$ theory. (See also important papers $[44,45]$.)

Original Klebanov-Polyakov conjecture [41] related large- $N$ limit of the $3 d$ boundary sigma-model with the action

$$
S=\frac{1}{2} \int d^{3} x\left(\partial_{\nu} \varphi_{i} \partial^{\nu} \varphi^{i}+\frac{\lambda}{N}\left(\varphi_{i} \varphi^{i}\right)^{2}\right)
$$

for scalar fields $\varphi^{i}$ in the vector representation of $O(N)(i=1,2, \ldots, N)$ with the particular HS gauge theories of [5], so-called $A$ and $B$-models, in the bulk. In [46] Klebanov-Polyakov conjecture was extended to the boundary vector models with Chern-Simons interaction where the Chern-Simons level was related to the free phase parameter in the models of [5]. Fermionic analogues of these conjectures were put forward in [47-49] and were partially checked in [50] (for more recent results see [51, 52]), suggesting a nontrivial duality between bosonic and fermionic boundary models, so-called bosonization, [53-55].

Some difficulties of the original analysis of HS holography originated from non-locality of the naive perturbative approach in HS theory leading to divergencies in the holographic tests [42]. In $[51,52,56]$ it has been checked that unfolded HS equations in the local frame of $[14,15,57]$ properly reproduce the anticipated holographic results including the general case of Chern-Simons boundary theory, thus opening a way towards further analysis of HS holography in the framework of the nonlinear HS system of [5].

$A d S_{4} / C F T_{3}$ HS duality was extended to $A d S_{3} / C F T_{2}$ holography by Gaberdiel and Gopakumar [58] (see [59] for a review) that has been extensively studied in the literature (see e.g. [60]-[69]). Elaborating on $A d S_{3} / C F T_{2}$ duality, Gaberdiel and Gopakumar arrived at the interesting conjecture on the relation between certain compactifications of String Theory and $3 d$ HS theories [70] as well as on the structure of stringy HS symmetry [71], further elaborated in [72]-[75] (see also [76]). Remarkably, symmetries of the string-like HS theories proposed in this paper exhibit interesting similarities with the GaberdielGopakumar construction.

The vector boundary models were recently extended to so-called tensor models [77] with the scalar and spinor fields $\varphi_{i_{1} i_{2} \ldots i_{p}}$ carrying several color indices (symmetrized and/or traceless or not). The Lagrangian for the so-called $O(N)^{3}$ boundary model is

$$
S=\frac{1}{2} \int d^{d} x\left(\partial_{\underline{n}} \varphi_{i j k} \partial^{\underline{n}} \varphi^{i j k}+g \varphi_{i j k} \varphi_{n m}^{i} \varphi^{j n} \varphi^{k m l}\right) .
$$

For $O(N)^{p}$ tensor models with any $p>1$ the interaction vertex is of order $p+1$. One can also consider bosonic and fermionic tensor models of $\mathrm{U}(N)^{p}$ and $U S P(2 N)^{p}$ types. Recently, these models have been extensively studied in the literature [78]-[81] in particular due to their relation [82] to the SYK model [83, 84], providing a new class of models possessing an interesting large $N$ regime [85]-[89]. This suggests in turn that they should admit some holographically dual description. The case of $p=2$ corresponds to models with matrixvalued fields like in $N=4 \mathrm{SYM}$ theory. In this case, the holographically dual theory is String Theory. 
To the best of our knowledge no models holographically dual to the tensor sigma-models with $p>2$ beyond the original one-dimensional SYK model [83]-[85] are available in the literature though ideas that there should be some HS holographic description analogous to that for the $O(N)$ model were expressed e.g. in [89, 90]. It is immediately clear that the HS model of [5] as well as its generalizations to three [8] and any [91] dimensions are not appropriate duals since the spectrum of $O(N)$ singlet operators of rank- $p$ tensor models increases with $p$ due to the presence of new multi-particle states $[78,81]$. The same time, the sigma-model form of the boundary theory suggests that a bulk dual model has to be of a HS type.

An obvious class of conformal boundary models holographically dual to tensorial HS theories in the bulk first discussed by Becaria and Tseytlin in [78] is provided by the free boundary conformal fields $\varphi_{i_{1}, i_{2}, \ldots i_{n}}$ with the tensorial currents of the type of the vertex in (1.2), i.e., $J=\varphi_{i j k} \varphi_{n m}^{i} \varphi^{j n} \varphi^{k m l}$, or similar. Analogously to the Klebanov-Polyakov conjecture [41] for vector models, we conjecture that the proposed Coxeter HS theories are holographically dual to such higher currents in the free boundary models. Moreover, the $A d S_{4}$ Coxeter HS models admit an extension to complex coupling constants that should be holographically dual to the $3 d$ boundary conformal models with Chern-Simons interactions analogous to the models considered in [46] for the vectorial HS holography. A subtlety of the holographic interpretation of the boundary tensor sigma-models is that their conformal structure analogous to that of critical sigma-model in the Klebanov-Polyakov conjecture [41] is not yet obvious. Note however that, as argued in [92], it is not a priory guaranteed that the boundary duals of the class of HS theories proposed in this paper are represented by local boundary sigma-models free from additional interactions via boundary HS conformal fields which may effectively induce nonlocal interactions on the boundary (see also [93]).

An important problem in the context of HS duality consists of the construction of so-called multi-particle HS theories containing multi-particle composite operators of the original HS theory as fundamental operators. In [94] a multi-particle extension of the usual HS symmetry was proposed, conjectured to underly an infinite extension of the conventional HS theory. The aim of this paper is to propose a class of generalized nonlinear HS systems to be associated with the tensor boundary sigma-models and their multi-particle extensions including the vector and tensor ones.

The proposed models are characterized by an integer $p$ analogous to the rank of the tensor $\varphi_{i_{1} i_{2} \ldots i_{p}}$ and have a much richer spectrum than usual HS theory of $[5,8,91]$. They are based on the deformed oscillator algebras found in [95-97] in the context of $p$-particle Calogero models, known as Cherednik algebras [98], which in turn are associated with various Coxeter groups. HS-like models of this class could be formulated long ago and potential relevance of the Cherednik algebra to HS theory was mentioned already in [97]. However, naive extension of this class was not formulated explicitly so far because the resulting spectra of states left no room for a massless spin-two state, i.e., graviton, not allowing the resulting models to fit into the standard paradigm of HS gravity. The same time, the naive extension to HS theories of this kind exhibits interesting features indicating their potential relevance as bulk duals of the tensor boundary sigma-models. 
In this paper we extend the construction of Cherednik algebras to framed Cherednik algebras in such a way that the related HS theories turn out to be free of the aforementioned difficulties, containing massless HS fields in the spectrum. This generalization was inspired by the construction of multi-particle algebras of [94] and, other way around, allows us to formulate nonlinear equations for the multi-particle HS theory. It is based on the $q^{\text {th }}$ direct product degree of a chosen rank- $p$ Coxeter group $\mathcal{C}$, thus containing two positive integers as free parameters: $p$ and $q$ are associated, respectively, with the tensor degree of the boundary model and the degree of multi-particle states. For instance $\mathcal{C}=A_{1} \sim B_{1}$ and $q=1$ or $q=\infty$ correspond to the conventional HS theory or its multi-particle extension, respectively. On the other hand, higher ranks $p$ of the Coxeter group $B_{p}$ are conjectured to be associated with the rank- $p$ tensor boundary models. Generalization to other Coxeter groups and root systems (for more detail on Coxeter groups see for instance [99]) is also possible and worth to be investigated. The case of $p=2, q=\infty$ is particularly appealing in connection with the HS interpretation of String Theory. The $B_{2}$-model possesses two coupling constants responsible for HS interactions in AdS background and stringy effects, respectively. It is tempting to speculate that this model can provide a realization of the most symmetric phase of String Theory. A possible HS symmetry breaking mechanism is also discussed in the concluding section of this paper.

The main idea of this work was to look for HS bulk models providing generalizations of the usual HS theory to models with richer spectrum that would qualitatively match the pattern of operators of the tensor boundary models and their multi-particle extensions. It should be stressed that the formal consistency and gauge symmetries along with simple physical conditions like, e.g., Lorentz covariance severely restrict possible higher-rank extensions of HS theories. The HS-like models based on framed Cherednik algebras provide a distinguished and, may be, exhaustive possibility for the construction of models fulfilling these properties.

The paper is organized as follows. We start with recalling the form of standard nonlinear HS equations in section 2. In section 3 we recall the construction of deformed oscillators that underlies the standard formulation of HS equations as well as its Cherednik extension associated with an arbitrary Coxeter root system. In section 4 we explain the difficulty of the naive extension of HS algebras via enlargement of the number of species of oscillators and introduce the notion of the framed oscillator algebra free of this difficulty. Then in section 5 we apply this idea to the construction of framed Cherednik algebras underlying the proper extension of HS equations to any Coxeter system presented in section 6 . Further extensions of the proposed systems to higher differential forms generating invariant functionals, color systems with Chan-Paton factors, and idempotents associated with brane-like dynamical systems in different dimensions are presented in sections 7, 8 and 9, respectively. Unitarity of the proposed models is discussed in section 10. Graded extension of the construction of multi-particle algebras of [94] along with the discussion of its different frames and new idempotent construction are presented in section 11. General properties of the proposed systems, their interpretation in the context of string-like and tensor-like HS systems and a possible HS symmetry breaking mechanism are discussed in section 12 where it is also argued that the proposed scheme allows one to interpret composite fields 
in the original space-time as elementary fields in higher dimensions. Section 13 contains brief conclusions.

\section{Standard higher-spin equations}

Nonlinear HS equations of $[5,8,91]$ are formulated in terms of three types of fields

$$
W(Z ; Y ; k \mid x)=d x^{\underline{n}} W_{\underline{n}}(Z ; Y ; k \mid x), \quad B(Z ; Y ; k \mid x), \quad S=d Z^{A} S_{A}(Z ; Y ; k \mid x) .
$$

Here $x^{\underline{n}}$ are space-time coordinates while the variables $Z^{A}$ and $Y^{A}(A, B, \ldots=1, \ldots M)$ are auxiliary coordinates acquiring different interpretation in the spinorial HS models of $[5,8]$ and vectorial HS model of [91]. $d Z^{A}$ are anticommuting $Z$-differentials

$$
d Z^{A} d Z^{B}=-d Z^{B} d Z^{A}, \quad d Z^{A} d x^{\underline{n}}=-d x^{\underline{n}} d Z^{A}, \quad d x^{\underline{m}} d x^{\underline{n}}=-d x^{\underline{n}} d x^{\underline{m}} .
$$

HS equations of $[5,8,91]$ have the form

$$
\begin{aligned}
& \mathrm{d} W+W * W=0, \\
& \mathrm{~d} B+W * B-B * W=0, \\
& \mathrm{~d} S+W * S+S * W=0, \\
& S * B=B * S, \\
& S * S=-i\left(d Z^{A} d Z_{A}+\left(d z^{\alpha} d z_{\alpha} F_{*}(B) * \kappa+\text { c.c. }\right)\right) .
\end{aligned}
$$

Here $\mathrm{d}=d x^{\underline{n}} \frac{\partial}{\partial d x^{\underline{n}}}$ is the space-time de Rham differential and $*$ denotes a model-dependent star product. $F_{*}(B)$ is some star-product function of the field $B$. Index $\alpha$ takes two values enumerating basis elements of some subspace of the space labelled by $A$. The complex conjugated term c.c. does not contribute in the real models of $[8,91]$ but has to be added in the $4 d$ model of [5]. $\kappa$ is an element of the algebra defined to commute with everything except for $d z^{\alpha}$ with which it anticommutes

$$
\kappa * d z^{\alpha}=-d z^{\alpha} * \kappa
$$

The associative star product $*$ acts on functions of two spinor variables

$$
(f * g)(Z ; Y)=\frac{1}{(2 \pi)^{4}} \int d^{4} U d^{4} V \exp \left[i U^{A} V^{B} C_{A B}\right] f(Z+U ; Y+U) g(Z-V ; Y+V),
$$

where $C_{A B}$ is a symplectic form and $U^{A}, V^{B}$ are real integration variables. It is normalized in such a way that 1 is a unit element of the star-product algebra, i.e., $f * 1=1 * f=f$. Star product (2.9) provides a particular realization of the Weyl algebra

$$
\left[Y_{A}, Y_{B}\right]_{*}=-\left[Z_{A}, Z_{B}\right]_{*}=2 i C_{A B}, \quad\left[Y_{A}, Z_{B}\right]_{*}=0
$$

$\left([a, b]_{*}=a * b-b * a\right)$ resulting from the normal ordering with respect to the elements

$$
b_{A}=\frac{1}{2 i}\left(Y_{A}-Z_{A}\right), \quad a_{A}=\frac{1}{2}\left(Y_{A}+Z_{A}\right),
$$


which satisfy

$$
\left[a_{A}, a_{B}\right]_{*}=\left[b_{A}, b_{B}\right]_{*}=0, \quad\left[a_{A}, b_{B}\right]_{*}=C_{A B} .
$$

The choice of the star-product realization (2.9) of the Weyl algebra is significant from many perspectives and, in particular, for the analysis of locality in [17, 18]. An important property of the star product (2.9) is that it admits the inner Klein operator

$$
\varkappa=\exp i Z_{\alpha} Y^{\alpha}
$$

which behaves as $(-1)^{N}$, where $N$ is the oscillator number operator, i.e.,

$$
\begin{gathered}
\varkappa * \varkappa=1, \\
\varkappa * f(Z ; Y)=f(\tilde{Z} ; \tilde{Y}) * \varkappa,
\end{gathered}
$$

where $\tilde{Z}^{A}=\left(-Z^{\alpha}, Z^{a}\right)$ for any $Z^{A}=\left(Z^{\alpha}, Z^{a}\right)$ with $Z^{a}$ being the rest components of $Z^{A}$. The Klein operator

$$
k=\kappa \varkappa,
$$

on which all fields (2.1) depend, anticommutes with $Y^{\alpha}, Z^{\alpha}$ and $d Z^{\alpha}$. Hence, $\kappa$ in eq. (2.7) is represented as

$$
\kappa=k \varkappa .
$$

(Note that the fields of the $4 d$ model of [5] also depend on the conjugated Klein operator $\bar{k}$.)

\section{Deformed oscillator algebras}

\subsection{Single oscillator}

The nontrivial part of the HS equations is represented by eqs. (2.6), (2.7). Since $B$ is covariantly constant by (2.4), and commutes with $S$ by (2.6) and $\kappa$ since it is a $d z$-independent zero-form, it behaves as a central element. Replacing $F_{*}(B)$ by a central element $\nu$ and discarding for a moment the complex conjugated term we observe that the nontrivial part of the HS equations has the form

$$
S * S=-i\left(d Z^{A} d Z_{A}+\nu * d z^{\alpha} d z_{\alpha} * \kappa\right), \quad \alpha=1,2 .
$$

This equation has many important interpretations for particular realizations of $S$, * and $\kappa$ (see e.g. $[6,10]$ ). The most important property in the context of this paper is that the r.h.s. of (3.1) commutes with $S$ despite of (2.8) since any trilinear combination of $d z^{\alpha}$ is zero because $\alpha=1,2$ and $d z^{\alpha}$ anticommute. It is this property that guarantees formal consistency of HS equations (2.3)-(2.7).

Eq. (3.1) induces commutation relations of the deformed oscillator algebra. Indeed, let $S=d z^{\alpha} q_{\alpha}+\ldots$, where ellipses denotes terms proportional to components of $d Z^{A}$ different from $d z^{\alpha}$, and

$$
\tilde{\nu}=\nu k,
$$

where

$$
k d z^{\alpha}=-d z^{\alpha} k, \quad k q_{\alpha}=-q_{\alpha} k, \quad k^{2}=1 .
$$


So defined $k$ and $\tilde{\nu}$ still commute with $S$ while, setting

$$
\varkappa:=\kappa k,
$$

eq. (3.1) implies deformed oscillator commutation relations in the form of [100]

$$
\left[q_{\alpha}, q_{\beta}\right]=-2 i \epsilon_{\alpha \beta}(1+\tilde{\nu} \varkappa), \quad \varkappa q_{\alpha}=-q_{\alpha} \varkappa, \quad \varkappa^{2}=1,
$$

where $\tilde{\nu}$ is central. The enveloping algebra $A q(\tilde{\nu})$ of these relations, which is the algebra of polynomials $f(q, \varkappa)$ with $\tilde{\nu}$ being a parameter, has the fundamental property [100] that

$$
t_{\alpha \beta}:=\frac{i}{4}\left\{q_{\alpha}, q_{\beta}\right\}
$$

generate $s p(2)$

$$
\left[t_{\alpha \beta}, t_{\gamma \delta}\right]=\epsilon_{\beta \gamma} t_{\alpha \delta}+\epsilon_{\beta \delta} t_{\alpha \gamma}+\epsilon_{\alpha \gamma} t_{\beta \delta}+\epsilon_{\alpha \delta} t_{\beta \gamma}
$$

rotating $q_{\alpha}$ as a $s p(2)$ vector

$$
\left[t_{\alpha \beta}, q_{\gamma}\right]=\epsilon_{\beta \gamma} q_{\alpha}+\epsilon_{\alpha \gamma} q_{\beta} .
$$

By these properties, the deformed oscillators (3.5) provide a representation-free realization of the Wigner oscillator [101] considered by many authors (see, e.g., [102-104]).

That the deformed oscillator algebra possesses the $s p(2)$ automorphism algebra implies that nonlinear HS equations (2.3)-(2.7) are also $s p(2)$ covariant. In $4 d$ HS theory of [5] and $3 d$ HS theory of [8] the respective $s p(2 \mid \mathbb{C})$ and $s p(2 \mid \mathbb{R})$ imply usual Lorentz covariance [6]. In vectorial models in any dimension of $[91], s p(2 \mid \mathbb{R})$ is a Howe dual algebra allowing to truncate away extra states from the spectrum.

\subsection{Many oscillators}

The deformed oscillator of section (3.1) can be interpreted as describing the relative motion in the two-body Calogero model. Extension to the $\mathcal{N}$-body Calogero model proposed in [9597] consist of $\mathcal{N}$ copies of oscillators $q_{\alpha i}$ with $\alpha=1,2, i=1, \ldots \mathcal{N}$ and the generators $K_{i j}$ of the symmetric group $S_{\mathcal{N}}$ that obey

$$
\begin{aligned}
K_{i j} q_{\alpha i} & =q_{\alpha j} K_{i j}, \\
K_{i j}=K_{j i}, \quad K_{i j} K_{i j} & =I, \quad K_{i j} K_{j l}=K_{i l} K_{i j}=K_{j l} K_{i l}
\end{aligned}
$$

(no summation over repeated indices), and $K_{i j}$ commutes with $q_{\alpha l}$ with $i \neq l, j \neq l$. Commutation relations of the oscillators $q_{\alpha l}$ have the form [95-97]

$$
\left[q_{\alpha n}, q_{\beta m}\right]=-i \epsilon_{\alpha \beta}\left(\delta_{n m}\left(2 I+\nu \sum_{l=1}^{\mathcal{N}} K_{l n}\right)-\nu K_{n m}\right) .
$$

Remarkably, this deformation still respects Jacobi identities since antisymmetrization over any three two-component indices gives zero as is most obvious from the Coxeter realization presented in section 3.3. 
From (3.11) it follows that the center of mass coordinates

$$
Q_{\alpha}:=\mathcal{N}^{-\frac{1}{2}} \sum_{n=1}^{\mathcal{N}} q_{\alpha n}
$$

have undeformed commutation relations with themselves and relative coordinates

$$
\left[Q_{\alpha}, Q_{\beta}\right]=-2 i \epsilon_{\alpha \beta}, \quad\left[Q_{\alpha}, q_{\beta n}-q_{\beta m}\right]=0 .
$$

The fundamental property of relations (3.11) is that the operators

$$
t_{\alpha \beta}:=\frac{i}{4} \sum_{n=1}^{\mathcal{N}}\left\{q_{\alpha n}, q_{\beta n}\right\}
$$

obey the $s p(2)$ commutation relations (3.7), properly rotating all indices $\alpha$ by virtue of

$$
\left[t_{\alpha \beta}, q_{\gamma n}\right]=\epsilon_{\beta \gamma} q_{\alpha n}+\epsilon_{\alpha \gamma} q_{\beta n} .
$$

Clearly, $t_{\alpha \beta}$ can be represented as a sum of $s p(2)$ generators acting separately on the center of mass and relative coordinates

$$
t_{\alpha \beta}=t_{\alpha \beta}^{c m}+t_{\alpha \beta}^{r e l}, \quad t_{\alpha \beta}^{c m}:=\frac{i}{4}\left\{Q_{\alpha}, Q_{\beta}\right\} .
$$

At $\mathcal{N}=2$, there is only one relative coordinate

$$
q_{\alpha}=\frac{1}{\sqrt{2}}\left(q_{\alpha 1}-q_{\beta 2}\right) .
$$

With $K_{12}=\varkappa(3.11)$ amounts to (3.5) which therefore describes the relative coordinate sector of the two-body Calogero model.

\subsection{Coxeter groups and Cherednik algebras}

A rank- $p$ Coxeter group $\mathcal{C}$ is generated by reflections with respect to a system of root vectors $\left\{v_{a}\right\}$ in a $p$-dimensional Euclidean vector space $V$ with the scalar product $(x, y) \in \mathbb{R}$, $x, y \in V$ (for more detail see [99]). An elementary reflection associated with the root vector $v_{a}$ acts on $x \in V$ as follows

$$
\mathcal{R}_{v_{a}} x^{i}=x^{i}-2 v_{a}^{i} \frac{\left(v_{a}, x\right)}{\left(v_{a}, v_{a}\right)}, \quad \mathcal{R}_{v_{a}}^{2}=I
$$

(no summation over $a$ ). $p$ is the rank of the Coxeter root system $\left\{v_{a}\right\}$. Note that $\mathcal{R}_{v_{a}}$ changes a sign of $v_{a}$

$$
\mathcal{R}_{v_{a}} v_{a}=-v_{a} .
$$

Let $q_{\alpha}^{n}(\alpha=1,2, n=1 ; \ldots, p)$ obey Heisenberg commutation relations

$$
\left[q_{\alpha}^{n}, q_{\beta}^{m}\right]=-2 i \epsilon_{\alpha \beta} \delta^{n m}, \quad q_{\alpha}^{n}=\delta^{n m} q_{m \alpha} .
$$


Cherednik deformation of the semidirect product of the Heisenberg algebra with the group algebra of $\mathcal{C}$ is

$$
\begin{aligned}
{\left[q_{\alpha}^{n}, q_{\beta}^{m}\right] } & =-i \epsilon_{\alpha \beta}\left(2 \delta^{n m}+\sum_{v \in \mathcal{R}} \nu(v) \frac{v^{n} v^{m}}{(v, v)} K_{v}\right), \\
K_{v} q_{\alpha}^{n} & =\mathcal{R}_{v}{ }^{n}{ }_{m} q_{\alpha}^{m} K_{v},
\end{aligned}
$$

where $K_{v}$ generate the Coxeter group, and the coupling constants $\nu(v)$ are invariants of $\mathcal{C}$ being constant on the conjugacy classes of root vectors under the action of $\mathcal{C}$. These relations generalize (3.11) from $A_{p}=S_{p+1}$ to any Coxeter group. By definition, the operators $K_{v}$ obey defining relation of the Coxeter group $\mathcal{C}$. In particular, each $K_{v}$ is involutive

$$
K_{v} K_{v}=I
$$

and since the reflections generated by $v$ and $-v$ coincide, the respective reflection generators are also identified

$$
K_{-v}=K_{v} .
$$

It is not difficult to check that the double commutator of $q_{\alpha}^{n}$ in (3.21) respects the Jacobi identities for any (not necessarily $\mathcal{C}$-invariant) $\nu(v)$. Indeed, the non-zero part of the triple commutator of $q_{\alpha}^{n}, q_{\beta}^{m}, q_{\gamma}^{k}$ resulting from (3.21) is proportional $v^{n} v^{m} v^{k}$ and hence contains the total antisymmetrization over three two-component indices $\alpha, \beta, \gamma$ giving zero. Covariance of relations (3.21) under the action of the Coxeter group itself demands $\nu(v)$ be $\mathcal{C}$-invariant.

In the sector of relative coordinates, the commutation relations (3.11) is a particular case of (3.21) for the $A_{p}$ root system

$$
v^{n m}=e^{n}-e^{m},
$$

where $e^{n}$ form an orthonormal frame in $\mathbb{R}^{p+1} . V$ is the $p$-dimensional subspace of relative coordinates in $\mathbb{R}^{p+1}$ spanned by $v^{n m}$.

Another important case of the Coxeter root system is $B_{p}$ with the roots

$$
R_{1}=\left\{ \pm e^{n} \quad 1 \leq n \leq p\right\}, \quad R_{2}=\left\{ \pm e^{n} \pm e^{m} \quad 1 \leq n<m \leq p\right\} .
$$

In addition to permutations, $B_{p}$ contains reflections of any basis axis in $V=R^{p}$ generated by $v_{ \pm}^{n}= \pm e^{n}$. In this case $R_{1}$ and $R_{2}$ form two conjugacy classes of $B_{p}$. Let $K_{n}, K_{n m}$ and $K_{n m}^{+}$be associated with $e^{n}, e^{n}-e^{m}$ and $e^{n}+e^{m}$, respectively.

The defining relations of $B_{p}$ are represented by relations (3.10) for the symmetric group generators $K_{n m}$ along with

$$
K_{n} K_{n m}=K_{n m} K_{m}, \quad K_{n} K_{n}=I, \quad K_{n m}^{+}=K_{n} K_{n m} K_{n}
$$

(generators $K_{n}, K_{n m}$ and $K_{n m}^{+}$with pairwise different indices commute).

The Coxeter group underlying $3 d$ HS theory of [8] is $A_{1} \sim B_{1}{ }^{1}$ The case of $B_{2}$ is also of great importance since it is argued in section 12 to be related to the string-like models.

\footnotetext{
${ }^{1}$ More precisely the Coxeter root system $A_{1} \sim C_{1}$ while the root systems $B_{p}$ and $C_{p}$ differ by the normalization of root vectors [99] having the same Coxeter group.
} 
For any Coxeter root system the generators

$$
t_{\alpha \beta}:=\frac{i}{4} \sum_{n=1}^{p}\left\{q_{\alpha}^{n}, q_{\beta}^{n}\right\}
$$

obey the $s p(2)$ commutation relations (3.7), properly rotating all indices $\alpha$ by virtue of

$$
\left[t_{\alpha \beta}, q_{\gamma}^{n}\right]=\epsilon_{\beta \gamma} q_{\alpha}^{n}+\epsilon_{\alpha \gamma} q_{\beta}^{n}
$$

as is easy to see using (3.22) and (3.19). This fact is of fundamental importance for HS theories.

\section{Higher-spin modules and framed oscillator algebras}

A natural way to allow a richer spectrum in the model including, in particular, mixedsymmetry massless and massive states (the latter are known to be present in String Theory [19]) known to allow consistent interactions at least in the lowest orders [105]-[111], is to let more species of oscillators in the model: $y_{\alpha} \rightarrow y_{\alpha}^{n}$. However, the construction of oscillator algebras with multiple species of oscillators as well as their Cherednik deformation recollected in section 3.3 cannot be directly applied to the construction of physically acceptable HS theories for the following reason.

Consider a HS algebra called for brevity $h s_{1}$ with the single set of oscillators (i.e., $p=1)$. It is well known $[23,112]$ that the Fock $h s_{1}$-module $F_{1}$ describes free boundary conformal fields [113, 114], i.e., Dirac singletons [40, 115-119]. Their conformal dimension is identified with the weight $h_{1}$ of the vacuum state in $F_{1}$ with respect to the dilatation operator $D$ represented by a bilinear of oscillators in $h s_{1}$

$$
D|0\rangle=h_{1}|0\rangle .
$$

(In the AdS unitary frame $D$ is replaced by the energy operator $E$. For details on the oscillator realization of semisimple Lie algebras and their modules see, e.g., [120].) Correspondingly, lowest weight representations of the naively extended algebras $h s_{p}$ built from $p$ copies of oscillators will have multiple weights in the respective Fock vacuum $F_{p}$,

$$
h_{p}=p h_{1},
$$

hence carrying higher lowest weights.

For $p=1$, elements of the tensor product $F_{1} \otimes F_{1}$ are interpreted either as conserved currents on the boundary or as massless fields in the bulk as was originally shown by Flato and Fronsdal [40]. However for $p>1$ the lowest weights of the submodules in $F_{p} \otimes F_{p}$ turn out to be too high to describe both conserved boundary currents and bulk massless fields. In particular, the $p>1$ modules have no room for a massless spin-two field, i.e., graviton. That the respective theory cannot contain gravity makes it impossible the construction of consistent HS models via allowing more oscillators, i.e., more generating elements of the star-product algebra. (Note that this argument does not apply to $3 d$ HS theories since in 
this case the graviton is topological [121, 122], carrying no local degrees of freedom, and, hence, having no nontrivial associated module of the space-time symmetry group. As a result, the construction of $3 d$ HS theories turns out to be far less restrictive than in $d>3$.)

Recently, this difficulty has been resolved in the construction of multi-particle algebra [10] defined as the universal enveloping algebra of the usual HS algebra, i.e., $\mathrm{U}\left(h s_{1}\right)$. From this definition it is clear that HS algebra-modules form modules over the multi-particle HS algebra as well. As observed in [10], an important difference between the construction of multi-particle algebra and oscillator algebras with many oscillators is due to appearance of independent unit elements associated with each copy of oscillators.

Namely, in the usual oscillator algebras the fundamental commutation relations are

$$
\left[q_{\alpha}^{n}, q_{\beta}^{m}\right]=2 i \delta^{n m} \epsilon_{\alpha \beta} I,
$$

where $I$ is the unit element of the algebra. In the multi-particle HS algebra these relations are replaced by

$$
\left[q_{\alpha}^{n}, q_{\beta}^{m}\right]=2 i \delta^{n m} \epsilon_{\alpha \beta} I_{n},
$$

where "units" $I_{n}$ assigned to the respective species of the oscillators obey

$$
I_{n} I_{n}=I_{n}, \quad I_{n} I_{m}=I_{m} I_{n} .
$$

Such algebras will be called framed oscillator algebras. Note that the framed oscillator algebras are nothing else as multiple tensor products of the original associative oscillator algebra with no color indices $n$ and $m$, i.e., of a single pair of oscillators while usual oscillator algebras (4.3) with the single unit element are their quotients.

The same time multi-particle HS algebra is unital being endowed with the unit element $I$ unrelated to $I_{n}$. Roughly speaking, $I$ is associated with the physical vacuum with no excited states while $I_{n}$ is associated with the minimal energy state in the sector of $n$ th particles.

An important feature of the multi-particle algebras is that their elements are (graded)symmetrized over elementary species. In that case, the only single-particle element built from $I_{n}$ is

$$
e=\sum_{i=1}^{p} I_{i} .
$$

As such $e$ becomes algebraically independent of individual $I_{n}$ with the consequence that its eigenvalues may be different from multiples of those for $I_{n}$ which may have no sense at all.

Technically, in the framed oscillator algebras, the problem with vacuum eigenvalues is avoided as follows. The "units" $I_{n}$ form a set of idempotents allowing to consider Fock-type modules $F_{p}^{i}$ generated from vacua $\left|0^{i}\right\rangle$ obeying

$$
\begin{aligned}
I_{j}\left|0^{i}\right\rangle & =\delta_{i}^{j}\left|0^{i}\right\rangle, \quad a_{-}^{j}\left|0^{i}\right\rangle=0, \\
a_{+}^{j}\left|0^{i}\right\rangle & =0 \quad i \neq j,
\end{aligned}
$$

where $a_{ \pm}^{i}$ are the creation and annihilation operators built from $q_{\alpha}^{i}$. Clearly, the modules of the generators $t_{\alpha \beta}$ formed by $F_{p}^{i}$ are equivalent to the $p=1$ Fock module $F_{1}$. Note that 
for usual oscillator algebras this construction is applicable to the oscillators with indices $\alpha, \beta$ taking any even number of values $2 M$ in which case $t_{\alpha \beta}(3.28)$ generate $s p(2 M)$.

Since higher-rank HS theories are anticipated to be associated with the Cherednik-like deformation of the oscillator algebras, to let them contain gravity it is necessary to find a framed extension of the Cherednik algebra having a room for units $I_{n}$.

\section{$5 \quad$ Framed Cherednik algebras}

\section{$5.1 A_{N}$}

To illustrate the idea let us first consider the $A_{p-1}$ system of section 3.2. In addition to $q_{\alpha n}$ and $K_{n m}$ with $n, m=1, \ldots p$, we introduce elements $I_{n}$ that obey

$$
\begin{aligned}
I_{n} I_{m} & =I_{m} I_{n}, & I_{n} I_{n} & =I_{n}, \\
I_{n} q_{\alpha n} & =q_{\alpha n} I_{n}=q_{\alpha n}, & I_{n} q_{\alpha m} & =q_{\alpha m} I_{n} .
\end{aligned}
$$

In presence of idempotents $I_{n}$, the proper modification of the deformed oscillator relations consistent with (5.2) is

$$
\left[q_{\alpha n}, q_{\beta m}\right]=-i \epsilon_{\alpha \beta}\left(\delta_{n m}\left(2 I_{n}+\nu \sum_{l=1}^{p} \hat{K}_{l n}\right)-\nu \hat{K}_{n m}\right),
$$

where

$$
\hat{K}_{n m}=I_{n} I_{m} K_{n m} .
$$

Note that operators $\hat{K}_{n m}$ obey all relations of the symmetric group $S_{p}$ except for the second relation in (3.10) replaced by

$$
\hat{K}_{n m} \hat{K}_{n m}=I_{n} I_{m}
$$

$\hat{K}_{n m}$ are demanded to obey the analogs of (3.9)

$$
\hat{K}_{i j} q_{\alpha i}=q_{\alpha j} \hat{K}_{i j}
$$

and to commute with all $I_{l}$

$$
I_{l} \hat{K}_{n m}=\hat{K}_{n m} I_{l} \quad \forall l, n, m .
$$

(Note that by (5.1) this is consistent with the naive expectation that $K_{n m} I_{m}=I_{n} K_{n m}$.) Also, the following relations held true

$$
I_{n} \hat{K}_{n m}=I_{m} \hat{K}_{n m}=\hat{K}_{n m} .
$$

It should be stressed that the unhatted generators of the symmetric group $K_{n m}$ do not appear in the construction of the framed Cherednik algebra. Relation (5.5) is not of the braid group since $I_{n} I_{m}$ is not invertible. Hence, $\hat{K}_{n m}$ do not generate a group algebra.

This modification guarantees consistency of defining relations (5.3). Namely, (5.7) implies consistency of (5.3) and (5.2) while the modification (5.5) of the symmetric group relations plays no role in the Jacobi identity check

$$
\left[q_{n \alpha},\left[q_{m \beta}, q_{k \gamma}\right]\right]+\text { cycle }=0 .
$$




\subsection{General Coxeter group}

Extension of commutation relations (3.21) to framed Cherednik algebra is

$$
\left[q_{\alpha}^{n}, q_{\beta}^{m}\right]=-i \epsilon_{\alpha \beta}\left(2 \delta^{n m} I_{n}+\sum_{v \in \mathcal{R}} \nu(v) \frac{v^{n} v^{m}}{(v, v)} \hat{K}_{v}\right),
$$

where $I_{n}$ are idempotents analogous to those of section 4 and

$$
\hat{K}_{v}:=K_{v} \prod I_{i_{1}(v)} \ldots I_{i_{k}(v)},
$$

where the labels $i_{1}(v), \ldots, i_{k}(v)$ enumerate those $I_{n}$ that carry labels affected by the reflection $\mathcal{R}_{v}$. For instance, in the case of $B_{p}, \hat{K}_{v_{i j}}=K_{v_{i j}} I_{i} I_{j}$ for the root $v_{i j}$ generating the permutation of $e_{i}$, and $e_{j}$ and $\hat{K}_{v_{i}}=K_{v_{i}} I_{i}$ for $v_{i}$ representing the reflection of $e_{i}$ of $B_{p}$.

It is important that framed Cherednik algebra still possesses inner $s p(2)$ automorphisms generated by

$$
t_{\alpha \beta}:=\frac{i}{4} \sum_{n=1}^{p}\left\{q_{\alpha}^{n}, q_{\beta}^{n}\right\} I_{n}
$$

obeying (3.29). Evidently,

$$
\left[t_{\alpha \beta}, I_{n}\right]=0 .
$$

Note that usual Cherednik algebra results from the framed one by quotioning out the ideal identifying all $I_{n}$ with the unit element of the algebra.

\section{Coxeter higher-spin equations}

\subsection{General scheme}

The idea is to let all $x$-dependent fields $W, S$ and $B$ depend on $p$ sets of variables enumerated by the label $n=1, \ldots p$, that include $Y_{A}^{n}, Z_{A}^{n}(A=1, \ldots M)$, idempotents $I_{n}$, anticommuting differentials $d Z_{n}^{A}$ and Klein-like operators $\hat{K}_{v}$ associated with all root vectors of a chosen Coxeter group $\mathcal{C}$ (at the convention $\hat{K}_{-v}=\hat{K}_{v}$ ). As usual in HS theory, the fields $W, S$ and $B$ are allowed to be valued in any associative algebra $A$ (see also section 8). To make contact with the tensorial boundary theory, it is useful to set $A=\left(M a t_{N}\right)^{p}$ with elements represented by tensorial matrices $a^{u_{1} \ldots u_{p}} v_{1} \ldots v_{p}, u_{i}, v_{i}=1 \ldots N$. Here $p$ is the tensor degree of the boundary model while $N$ is the number of values of color indices with respect to which the $N \rightarrow \infty$ limit has to be taken on the boundary. In this limit the leading contribution should be represented by the colorless HS theory in the bulk.

The field equations associated with Cherednik algebra (3.21) are formulated in terms of the star product analogous to (2.9)

$$
\begin{aligned}
(f * g)(Z ; Y ; I)=\frac{1}{(2 \pi)^{p M}} \int d^{p M} S d^{p M} T \exp \left[i S_{n}^{A} T_{m}^{B} \delta^{n m} C_{A B}\right] & \\
& \times f\left(Z_{i}+I_{i} S_{i} ; Y_{i}+I_{i} S_{i} ; I\right) g(Z-T ; Y+T ; I)
\end{aligned}
$$

with central elements $I_{n}$ obeying relations

$$
I_{n} * Y_{A}^{n}=Y_{A}^{n} * I_{n}=Y_{A}^{n}, \quad I_{n} * Z_{A}^{n}=Z_{A}^{n} * I_{n}=Z_{A}^{n}, \quad I_{n} * I_{n}=I_{n} .
$$


With this definition star product (6.1) implies

$$
\left[Y_{A}^{n}, Y_{B}^{m}\right]_{*}=-\left[Z_{A}^{n}, Z_{B}^{m}\right]_{*}=2 i C_{A B} \delta^{n m} I_{n}, \quad\left[Y_{A}^{n}, Z_{B}^{m}\right]_{*}=0 .
$$

Analogously to eq. (2.13) this star product admits inner Klein operators $\varkappa_{v}$ associated with the root vectors $v$

$$
\varkappa_{v}:=\exp i \frac{v^{n} v^{m} Z_{\alpha n} Y^{\alpha}{ }_{m}}{(v, v)}
$$

and any subset of indices $\alpha$ among $A$ such that $\operatorname{det}\left|C_{\alpha \beta}\right| \neq 0$. It is straightforward to see that the Klein operators $\varkappa_{v}$ generate the star-product realization of the Coxeter group via

$$
\varkappa_{v} * q_{\alpha}^{n}=\mathcal{R}_{v}{ }^{n}{ }_{m} q_{\alpha}^{m} * \varkappa_{v}, \quad q_{\alpha}^{n}=Y_{\alpha}^{n}, Z_{\alpha}^{n} .
$$

Nonlinear equations for the generalized HS theory associated with the Coxeter group $\mathcal{C}$ are

$$
\begin{aligned}
& \mathrm{d} W+W * W=0, \\
& \mathrm{~d} B+W * B-B * W=0, \\
& \mathrm{~d} S+W * S+S * W=0, \\
& S * B=B * S, \\
& S * S=-i\left(d Z^{A n} d Z_{A n}+\sum_{i} \sum_{v \in \mathcal{R}_{i}} F_{i *}(B) \frac{d Z_{n}^{\alpha} v^{n} d Z_{\alpha m} v^{m}}{(v, v)} * \hat{\kappa}_{v}\right),
\end{aligned}
$$

where $\hat{\kappa}_{v}$ act trivially on (i.e., commute with) all elements except for $d Z_{\alpha n}$ on which they act in the standard fashion

$$
\hat{\kappa}_{v} * d Z_{\alpha}^{n}=\mathcal{R}_{v}{ }^{n}{ }_{m} d Z_{\alpha}^{m} * \hat{\kappa}_{v} .
$$

$F_{i *}(B)$ is any star-product function of the zero-form $B$ on the conjugacy classes $R_{i}$ of $\mathcal{C}$. For instance in the important case of $B_{p}$ equation (6.10) reads as

$$
\begin{aligned}
S * S=-i\left(d Z_{A n} d Z^{A n}+\sum_{v \in \mathcal{R}_{1}}\right. & F_{1 *}(B) \frac{d Z_{n}^{\alpha} v^{n} d Z_{\alpha m} v^{m}}{(v, v)} * \hat{\kappa}_{v} \\
& \left.+\sum_{v \in \mathcal{R}_{2}} F_{2 *}(B) \frac{d Z_{n}^{\alpha} v^{n} d Z_{\alpha m} v^{m}}{(v, v)} * \hat{\kappa}_{v}\right)
\end{aligned}
$$

with arbitrary $F_{1 *}(B)$ and $F_{2 *}(B)$. In particular, one can set $F_{2 *}(B)=0$ keeping $F_{1 *}(B)=$ $\eta B$. In the case of $B_{1}$ this gives usual HS equations. $F_{2 *}(B)$ can be nonzero for the $B_{p^{-}}$ HS models at $p \geq 2$. As discussed in more detail in section 12 , the roles of the coupling constants contained in $F_{1 *}(B)$ and $F_{2 *}(B)$ are different: the former are responsible for the HS features of the model in AdS while the latter for the stringy and, more generally, tensorial ones.

Perturbative analysis is performed around the vacuum solution

$$
B=0, \quad S_{0}=d Z^{A n} Z_{A n}, \quad W=W_{0}(Y \mid x),
$$


where $W_{0}(Y \mid x)$ is some solution to (6.6) that usually is taken to describe AdS space-time. (In the $3 d$ models of [8] the solution with $B=$ const is also important.)

The realization of $\hat{\kappa}_{v}$ can be different in different models and usually needs introduction of the outer Klein operators $\hat{k}_{v}$ that, by definition, obey (3.22) with $q=Y_{\alpha n}, Z_{\alpha n}$ and $d Z_{\alpha n}$, so that $\hat{\kappa}_{v}=\varkappa_{v} \hat{k}_{v}$ only affects the differentials $d Z_{\alpha n}$.

Equations (6.6)-(6.10) are formally consistent since relations (3.21) respect the Jacobi identities which in terms of the field equations are fulfilled due to the property that the r.h.s. of (6.10) is central. That HS-type equations can be consistently formulated based on Coxeter root systems was clear long ago. (This was mentioned already in [97].) However, such equations were never discussed in the literature so far since their interpretation in the standard HS paradigm was not easy as discussed in section 4 unless the construction is extended to the framed Cherednik algebras proposed in this paper.

Multi-particle extensions of the Coxeter HS systems are associated with the semisimple Coxeter groups. The simplest option is associated with the Coxeter root system $B_{p}^{\mathcal{N}}$ that consists of the product of $\mathcal{N} B_{p}$ systems

$$
B_{p}^{\mathcal{N}}:=\underbrace{B_{p} \times B_{p} \times \ldots}_{\mathcal{N}} .
$$

The limit $\mathcal{N} \rightarrow \infty$ along with the graded symmetrization of the product factors expressing the spin-statistics relation (for more detail see section 11) is in many respects most natural.

One can consider reductions of the Coxeter HS theories by restricting all fields to invariants of some group $S$ of automorphisms of $\mathcal{C}$. A natural choice is some subgroup $S=S_{p} \times S_{\mathcal{N}}$ with $S_{p} \in B_{p}$ and $S_{\mathcal{N}}$ exchanging the product factors in (6.14). The latter reduction is possible in the case with all functions $F_{i}(B)$ associated with different product factors in $\mathcal{C}$ equal to each other. Restriction to invariants of $S$ may be important for taking the $\mathcal{N} \rightarrow \infty$ limit.

The choice of $S$ containing the full symmetric group $S_{\mathcal{N}=\infty}$ is distinguished from various perspectives. If $S=S_{\infty}$, the resulting algebra is the (graded symmetric) multi-particle algebra $M(h(\mathcal{C}))$ of [94] of the HS algebra $h(\mathcal{C})$ associated with the $\mathcal{C}$-framed Cherednik algebra. As explained in [94], $M(h(\mathcal{C}))$ is isomorphic to the universal enveloping algebra of $h(\mathcal{C})$ (see also section 11). This implies in particular that $M(h(\mathcal{C})$ ) is a Hopf algebra. Analogously, if $S=S_{\infty} \times G$ with $G$ being some subgroup of the simple Coxeter group $\mathcal{C}$, the resulting multi-particle algebra is $M\left(h_{G}(\mathcal{C})\right)$ where $h_{G}(\mathcal{C})$ is the subgroup of invariants of $G$ in $h(\mathcal{C})$. Clearly, $M\left(h_{G}(\mathcal{C})\right)$ is also a Hopf algebra.

As discussed in section 12, the particularly important case of $B_{2}^{\infty}$ multi-particle HS model associated with the $\operatorname{sym}\left(B_{2} \times B_{2} \times \ldots\right)$ system, where the graded symmetrization is with respect to all elementary $B_{2}$ factors, is anticipated to represent a stringy HS model.

Now we are in a position to specify different types of the HS models resulting from the proposed construction.

\subsection{Spinor models}

Spinor HS models were formulated in three [8] and four [5] dimensions. Naively, these models may look being too far from Superstring living in 10 dimensions [19]. This is 
indeed true for the original $3 d$ and $4 d$ HS models but may not be true for their multiparticle extensions. The point is that, as emphasized in [123, 124], the multi-particle states of a lower-dimensional model can be identified with elementary states in an appropriate higher-dimensional theory. Such interpretation turns out to be most natural within the matrix-space approach to massless field theories elaborated in [114, 125-127] (see also [92] and recent reviews $[128,129]$ ). Moreover, the maximal space-time where such models admit interpretation in terms of local fields is ten-dimensional [125-127] which is just the superstring space-time dimension. (It would be interesting to see whether this phenomenon is related to the twistor-like transform in ten dimensions introduced by Witten in [130].) From this perspective the original $3 d$ and $4 d$ theories can be interpreted as certain branes in the ten-dimensional theory [126] with the $3 d$ HS model serving as an elementary brick from which the others are composed (see also section 12.2).

\subsection{1 $3 d$ Coxeter higher-spin models}

The $3 d$ Coxeter extension of the models of [8] is straightforward. It still needs introduction of two Clifford elements $\psi_{1,2}$ as in [8] to induce the doubling of the spectrum of fields as is standard in $3 d$ gravity [121, 122] and HS theory [131]. The indices $A$ and $\alpha$ coincide, i.e., $A$ takes just two values representing a $3 d$ spinor index. $s p(2)$ (5.12) represents the local Lorentz symmetry. For definiteness we consider the case of the Coxeter group $B_{p}$ which seems to be most appropriate for HS applications. Extension to other Coxeter groups is straightforward.

The multi-particle extension of the $3 d$ Coxeter HS theories results from endowing all variables with the additional flavour index $a=1, \ldots, \mathcal{N}$

$$
I_{n}, z_{\alpha n}, y_{\alpha n}, d z_{\alpha n}, \psi_{1,2}, \hat{\kappa}_{n}, \hat{\kappa}_{n m} \quad \longrightarrow \quad I_{n}^{a}, z_{\alpha n}^{a}, y_{\alpha n}^{a}, d z_{\alpha n}^{a}, \psi_{1,2}^{a}, \hat{\kappa}_{n}^{a}, \hat{\kappa}_{n m}^{a}
$$

One can either consider different functions $F_{1,2 *}^{a}(B)$ or to keep them all equal (i.e., $a$ independent) to allow the $S_{\mathcal{N}}$ reduction. In the latter case HS equations (6.12) read as

$$
\begin{aligned}
S * S= & -i \sum_{a=1}^{\mathcal{N}} \sum_{n=1}^{p}\left(d z_{\alpha n}^{a} d z^{\alpha n a}+F_{1 *}(B) * d z_{n}^{\alpha a} d z_{\alpha n}^{a} * \hat{\kappa}_{n}\right. \\
& \left.+2 F_{2 *}(B) * \sum_{m=1}^{p}\left(d z_{n}^{\alpha a}\left(d z_{\alpha n}^{a}-d z_{\alpha m}^{a}\right) * \hat{\kappa}_{n m}^{a}+d z_{n}^{\alpha a}\left(d z_{\alpha n}^{a}+d z_{\alpha m}^{a}\right) \hat{\kappa}_{n}^{a} * \hat{\kappa}_{n m}^{a} * \hat{\kappa}_{n}^{a}\right)\right)
\end{aligned}
$$

where $\kappa_{n}$ and $\kappa_{n m}$ are the generators of the Coxeter group $B_{p}$, that act only on $d z_{\alpha n}^{\alpha}$. The resulting model contains both bosons and fermions for any $\mathcal{N}$ including the $\mathcal{N}=1 B_{p}$-HS theory. The master fields are demanded to be graded symmetric under the exchange of odd combinations of spinors $z_{\alpha n}^{a}, y_{\alpha n}^{a}$ at different $a$ (see also section 11).

Further reductions can be performed with $S=S_{p}$ and/or $S=B_{p}$. Since fermions are represented by odd functions of $y$ the latter choice leads to a bosonic reduced model.

In the graded symmetrically reduced model usual HS gauge fields are represented by the $z$-independent one-form part $\sum_{i, a}^{p, \mathcal{N}} \omega\left(y_{i}^{a}, \psi_{1 i}^{a}, \hat{k}_{i}^{a} \mid x\right) * I_{i}^{a}$ of $W$ with $y_{\alpha i}^{a}$ anticommuting at different $a$. In particular, $A d S_{3}$ background fields belong to this field (for more detail see [8].) 
Massless matter fields are represented by the $z$-independent part $\sum_{i, a} C\left(y_{i}^{a}, \psi_{1 i}^{a}, \hat{k}_{i}^{a} \mid x\right) * \psi_{2 i}^{a}$ of the zero-form $B$. Indeed, due to the presence of idempotents $I_{i}^{a}$ (recall that $\psi_{2 i}^{a}=\psi_{2 i}^{a} * I_{i}^{a}$ ) these obey the same unfolded field equations as the massless fields of [8]. In the absence of $I_{i}^{a}$ the additional terms would contribute changing the pattern of the equations in agreement with the discussion of section 4 . In the framed system such terms are uplifted to the equations on the two-particle fields containing pairs of units $I_{i_{1}}^{a_{1}} I_{i_{2}}^{a_{2}}$.

As usual in HS theory $[5,6]$, one can consider models with fields valued in matrix algebras i.e., carrying Chan-Paton-like matrix indices. Upon imposing appropriate reality conditions the respective HS models possess $\mathrm{U}(n)$ gauge symmetry in the spin-one sector. Following the construction of $[8,139]$ (see also [6]) the resulting models can be truncated to those with $O(n)$ and $\operatorname{Sp}(2 m)$ spin-one gauge groups. (See also section 8.)

\subsection{2 $4 d$ Coxeter higher-spin models}

In the Minkowski signature $4 d$ Coxeter HS model, $A=\alpha, \dot{\alpha}$ and one introduces two mutually commuting conjugated algebras generated by the left and right elements $y_{\alpha}^{n}, z_{\alpha}^{n}, \hat{k}_{v}$ and $\bar{y}_{\dot{\alpha}}^{n}, \bar{z}_{\dot{\alpha}}^{n}, \hat{\bar{k}}_{\bar{v}}$, respectively, on which all fields $W, S$ and $B$ depend. In the $4 d B_{p}$-HS system, equation (6.12) is

$$
\begin{array}{r}
S * S=-i\left(d z_{A n} d z^{A n}+\sum_{v \in \mathcal{R}_{1}} F_{1 *}(B) \frac{d z_{n}^{\alpha} v^{n} d z_{\alpha m} v^{m}}{(v, v)} * \hat{\kappa}_{v}+\sum_{v \in \mathcal{R}_{2}} F_{2 *}(B) \frac{d z_{n}^{\alpha} v^{n} d z_{\alpha m} v^{m}}{(v, v)} * \hat{\kappa}_{v}\right. \\
\left.+\sum_{\bar{v} \in \mathcal{R}_{1}} \bar{F}_{1 *}(B) \frac{d \bar{z}_{n}^{\dot{\alpha}} \bar{v}^{n} d \bar{z}_{\dot{\alpha} m} \bar{v}^{m}}{(\bar{v}, \bar{v})} * \hat{\kappa}_{\bar{v}}+\sum_{\bar{v} \in \mathcal{R}_{2}} \bar{F}_{2 *}(B) \frac{d \bar{z}_{n}^{\dot{\alpha}} \bar{v}^{n} d \bar{z}_{\dot{\alpha} m} \bar{v}^{m}}{(\bar{v}, \bar{v})} * \hat{\kappa}_{\bar{v}}\right) \cdot(6.17)
\end{array}
$$

In fact, there are several options for the higher-rank extension of the $4 d$ HS theory. The simplest $\left(B_{p} \times B_{p}\right)^{\prime}$-option is with identified idempotents $I_{n}=\bar{I}_{n}$ associated with the left and right oscillators $y_{\alpha}^{n}, z_{\alpha}^{n}$ and $\bar{y}_{\dot{\alpha}}^{n}, \bar{z}_{\dot{\alpha}}^{n}$. We will call this HS model class $I$. Recall that by (5.7) both $I_{n}$ and $\bar{I}_{n}$ commute with the Klein operators $\hat{k}$ and $\bar{k}$. Hence $I_{n}-\bar{I}_{n}$ generates an ideal $\mathcal{I}$ of the $B_{p} \times B_{p}$ system with independent $I_{n}$ and $\bar{I}_{n}$. The $\left(B_{p} \times B_{p}\right)^{\prime}$ system is $\left(B_{p} \times B_{p}\right) / \mathcal{I}$. In this model the lowest states are associated with $4 d$ massless fields represented by functions of a single copy of oscillators $y_{\alpha}^{n}, z_{\alpha}^{n}, \bar{y}_{\dot{\alpha}}^{n}, \bar{z}_{\dot{\alpha}}^{n}$ and $I_{n}$. For instance, in the graded symmetrized case the massless fields are described by the $Z$-independent parts $\omega$ and $C$ of $W$ and $B$, respectively

$$
\begin{aligned}
\omega & =\sum_{i, a}^{p, \mathcal{N}} \omega\left(y_{i}^{a}, \hat{k}_{i}^{a} ; \bar{y}_{i}^{a}, \hat{\bar{k}}_{i}^{a} \mid x\right) * I_{i}^{a} \quad \omega\left(y_{i}^{a}, \hat{k}_{i}^{a} ; \bar{y}_{i}^{a}, \hat{\bar{k}}_{i}^{a} \mid x\right)=\omega\left(y_{i}^{a},-\hat{k}_{i}^{a} ; \bar{y}_{i}^{a},-\hat{\bar{k}}_{i}^{a} \mid x\right), \\
C & =\sum_{i, a}^{p, \mathcal{N}} C\left(y_{i}^{a}, \hat{k}_{i}^{a} ; \bar{y}_{i}^{a}, \hat{\bar{k}}_{i}^{a} \mid x\right) * I_{i}^{a} \quad C\left(y_{i}^{a}, \hat{k}_{i}^{a} ; \bar{y}_{i}^{a}, \hat{\bar{k}}_{i}^{a} \mid x\right)=-C\left(y_{i}^{a},-\hat{k}_{i}^{a} ; \bar{y}_{i}^{a},-\hat{\bar{k}}_{i}^{a} \mid x\right) .
\end{aligned}
$$

The $4 d$ model with different $I_{n}^{a} \neq \bar{I}_{n}^{a}$ which we call class $I I$ is a particular real form of the complexified $3 d\left(B_{p}\right)^{2}$ system which construction is fully analogous to the $s l_{2}(\mathbb{C})$ realization of the $4 d$ Lorentz algebra. In fact this is a simplest manifestation of the fact discussed in the beginning of section 6.2 that tensoring of the lower-dimensional models 
can give higher-dimensional ones. Though this phenomenon is less straightforward for the higher tensor products since higher-dimensional Lorentz algebras are not real forms of the direct sums of a number of $s l_{2}(\mathbb{C})$ the results of [125]-[129] suggest that a proper interpretation can be available in a certain limit in which the contribution of (delocalized) branes associated with the lower rank (dimensional) models can be discarded. In this model, the lower-rank states start with $\omega$ and $C$ that depend either only on $y_{n}^{a}, \hat{k}_{n}^{a}$ or only on $\bar{y}_{n}^{a}, \hat{\bar{k}}_{n}^{a}$. These states are analogous to the $3 d$ massless states to be identified with the boundary states in the $A d S_{4} / C F T_{3}$ holography once the background (i.e., vacuum) fields are chosen appropriately. This opens an interesting possibility for describing both bulk and boundary degrees of freedom in the same model. A natural framework for the realization of this option suggested by the approach of [92] is described in section 9 .

Finally, there is an interesting further extension of the $4 d$ HS system to the $B_{2 p}(\mathbb{C})$ model, referred to as class $I I I$ which is a specific real form of the complexified $B_{2 p} 3 d$

system where the $\hat{K}_{i \bar{j}}$ reflection that exchanges the spinor variables of opposite chiralities is antilinear to be compatible with the fact that the dotted and undotted spinors exchanged by $\hat{K}_{i \bar{j}}$ are complex conjugated. Note that this leads to a parametric freedom in the model via the phase parameter $\varphi$ in the complex structure relations

$$
\hat{K}_{i \bar{j}}=\exp i \varphi \hat{\bar{K}}_{\overline{i j}} .
$$

For the $4 d$ models the natural choice of the symmetric reduction is with $S=S_{p}$ being the diagonal subgroup of $S_{p} \times \bar{S}_{p}$ in the left and right sector, allowing to keep the frame-like field

$$
h^{\alpha \dot{\alpha}} \sum_{n=1}^{p} y_{n \alpha} \bar{y}_{n \dot{\alpha}}
$$

$S$-invariant, as well as its further extension to $S=S_{p} \times S_{\mathcal{N}}$ in the multi-particle case.

\subsection{Vector Coxeter higher-spin models in any dimension}

Vector HS model in $d$ dimensions of [91] is formulated in terms of the oscillators $Z_{n}^{A}, Y_{n}^{A}$ with the double index $A=\nu, \alpha$ where $\nu=0, \ldots d$ is the vector index of the $d$-dimensional AdS algebra $o(d-1,2)$ and $\alpha=1,2$ is the vector index of $s p(2)$ with the symplectic form $\epsilon_{\alpha \beta}$ so that

$$
C_{A B}=\eta_{\nu \mu} \epsilon_{\alpha \beta}, \quad A=\nu, \alpha, \quad B=\mu, \beta,
$$

where $\eta_{\nu \mu}$ is the (x-independent) $o(d-1,2)$-invariant fiber metric. The index $\nu$ is decomposed into $\nu=\left(\nu^{\|}, \nu^{\perp}\right)$ where $\nu^{\perp}=0, \ldots, d-1$ labels the Lorentz components transforming as a $o(d-1,1)$ vector while $\nu^{\|}$, taking one value, labels the Lorentz-invariant component. The index $\alpha$ of the nontrivial part of eq. (6.10) is identified with $\nu=\left(\nu^{\|}, \alpha\right)$ (i.e., with the $d+1$ th component of the $o(d-1,2)$ vector index $\nu$ in $A=\nu, \alpha)$ and still takes two values.

The original HS system of [91] is formulated in terms of the star product

$$
(f * g)(Z, Y)=\frac{1}{\pi^{2(d+1)}} \int d S d T e^{-2 S_{\alpha}^{A} T_{A}^{\alpha}} f(Z+S, Y+S) g(Z-T, Y+T),
$$


which gives rise to the commutation relations

$$
\left[Z_{\alpha}^{\nu}, Z_{\beta}^{\mu}\right]_{*}=-\epsilon_{\alpha \beta} \eta^{\nu \mu}, \quad\left[Y_{\alpha}^{\nu}, Y_{\beta}^{\mu}\right]_{*}=\epsilon_{\alpha \beta} \eta^{\nu \mu}, \quad\left[Y_{\alpha}^{\nu}, Z_{\beta}^{\mu}\right]_{*}=0
$$

The analogue of eq. (2.7) has slightly different normalization

$$
S * S=-\frac{1}{2}\left(d Z_{A}^{\alpha} d Z_{\alpha}^{A}+4 d Z_{\nu \| \alpha} d Z_{\nu \|}^{\alpha} B * \kappa\right) .
$$

$\kappa$ is the product of the inner Klein operator $\varkappa$ and the outer Klein operator $k$ that anticommutes with all $\nu^{\|}$components but commutes with the $\nu^{\perp}$ Lorentz components.

The nontrivially deformed part of (6.24) is of the $B_{1}$ type. To extend the $s p(2)$ algebra of automorphisms of the $B_{1}$ Cherednik algebra to the full $s p(2)$ rotations of $(\nu, \alpha)$ with any $\nu$, for $\nu \neq d$ the action of $s p(2)$ is generated by the $s p(2)$ of undeformed oscillators. The full $s p(2)$ generators are

$$
t_{\alpha \beta}:=-\frac{1}{2} \sum_{n=1}^{\mathcal{N}}\left\{S_{\nu \alpha}^{n}, S_{\mu \beta}^{n} \eta^{\nu \mu}\right\}_{*} .
$$

$t_{\alpha \beta}^{\text {tot }}$ is the $s p(2)$ generator acting on all indices $\alpha, \beta$ including those of differentials $d Z_{\nu \alpha}$. In addition, in the model of [91] it is demanded that the generator

$$
t_{\alpha \beta}^{\text {int }}:=t_{\alpha \beta}^{\text {tot }}-t_{\alpha \beta}
$$

obeys relations

$$
\mathrm{d} t_{\alpha \beta}^{\mathrm{int}}-\left[t_{\alpha \beta}^{\mathrm{int}}, W\right]_{*}=0, \quad\left[t_{\alpha \beta}^{\mathrm{int}}, S\right]_{*}=0, \quad\left[t_{\alpha \beta}^{\mathrm{int}}, B\right]_{*}=0
$$

(that restricts the spectrum of dynamical fields to two-row rectangular Young diagrams of $o(d-1,2))$ and that the fields in the ideal formed by the elements of the form $t_{\alpha \beta}^{\text {int }} * f^{\alpha \beta}$ or $f^{\alpha \beta} * t_{\alpha \beta}^{\text {int }}$ have to be factored out, that restricts the spectrum of dynamical fields to traceless two-row rectangular Young diagrams of $o(d-1,2)$ appropriate for the description of genuine massless HS fields in the unfolded formalism [132, 133].

The Coxeter extension needs several species of oscillators. Here however is a subtlety that, as in the simplest $B_{1}$ case of [91], the Lorentz components $\nu^{\perp}$ of the $A d S_{d}$ vectors should be treated differently from the $d+1$ th components $\nu^{\|}$. The latter are supposed to be affected by the action of the Coxeter reflections while the former are not to preserve manifest Lorentz covariance. (Note that the latter condition is needed in the Lorentz covariant setup but may be relaxed in a more general situation.) Naively, this suggests that, to use the framed Coxeter algebra construction, one has to introduce different idempotent elements for the $d+1$ th components of oscillators and the Lorentz ones. This is however not necessary since Coxeter HS equations involve hatted Klein operators that obey (5.7) allowing to use $o(d-1,2)$-covariant commutation relations

$$
\left[Y_{n}^{\nu \alpha}, Y_{m}^{\mu \beta}\right]=\delta_{n m} \epsilon^{\alpha \beta} \eta^{\nu \mu} I_{n} .
$$

In terms of these oscillators, the $o(d-1,2)$ algebra is realized by the generators

$$
T^{\nu \mu}=-\frac{1}{2} \epsilon^{\alpha \beta} \sum_{n=1}^{\mathcal{N}} Y_{n \alpha}^{\nu} Y_{n \beta}^{\mu} .
$$


The $s p(2)$ generators are

$$
t_{\alpha \beta}:=-\frac{1}{2} \eta_{\nu \mu} \sum_{n=1}^{\mathcal{N}}\left\{S_{n \alpha}^{\nu}, S_{n \beta}^{\mu}\right\}_{*} .
$$

The generators $t^{\text {int }}$ and $t^{\text {tot }}$ are defined as in the original model using (6.26).

Analogously to the original HS model, one imposes the $s p(2)$ invariance condition followed by the factorization condition $t_{\alpha \beta}^{\text {int }} * f^{\alpha \beta} \sim f^{\alpha \beta} * t_{\alpha \beta}^{\text {int }} \sim 0$. An important remaining question is whether this is sufficient to remove all unnecessary states from the general Coxeter HS model making it unitary. The natural choice of the symmetric reduction is with the symmetric group $S_{p}$ that acts on all indices $n$ of $Y_{A n}, Z_{A n}$ and $d Z_{A n}$ irrespectively of the value of $A$ and/or $S_{\mathcal{N}}$ in the multi-particle extension.

Finally let us note that, in agreement with the analysis of [134], it is natural to expect the existence of another HS model in $d$ dimensions which is holographically dual to free conformal fermions on the boundary, as well as the further supersymmetric extension of the bosonic and fermionic models. Though the explicit form of the nonlinear field equations was not presented so far, we anticipate that it can be elaborated along the lines of [91]. (Recently, some low-order results in the construction of the fermionic model were presented in [135].) Such models will also generate a class of Coxeter HS theories in $d$ dimensions.

\section{Higher forms and invariants}

HS theories admit an important extension to higher differential forms along the lines of [136]. To this end, it is convenient to unify the $d Z$ - and $d x$-one-forms into the master field

$$
\mathcal{W}_{1}=\mathrm{d}+d x^{\underline{\underline{n}}} W_{\underline{\underline{n}}}(Z ; Y ; \hat{K} \mid x)+d Z^{A} S_{A}(Z ; Y ; \hat{K} \mid x), \quad \mathrm{d}=d x^{\underline{\underline{n}}} \frac{\partial}{\partial x^{\underline{n}}},
$$

extending it further to all higher differential forms of odd total degrees with respect to both $d Z$ and $d x$,

$$
\mathcal{W}=\sum_{p=1,3, \ldots} \mathcal{W}_{p}
$$

Analogously, the zero-form master field $B$ is extended to the field $\mathcal{B}$ containing differential forms of all total even degrees

$$
\mathcal{B}=\sum_{p=0,2, \ldots} \mathcal{B}_{p}, \quad \mathcal{B}_{0}=B
$$

In these terms the appropriately extended system (6.6)-(6.10) takes the form

$$
\begin{aligned}
\mathcal{W} * \mathcal{W} & =-i\left(d Z^{A n} d Z_{A n}+F_{*}\left(\mathcal{B}, \gamma_{i}\right)\right) \\
{[\mathcal{W}, \mathcal{B}]_{*} } & =0
\end{aligned}
$$

where

$$
\gamma_{i}=\sum_{v \in \mathcal{R}_{i}} \frac{d Z_{n}^{\alpha} v^{n} d Z_{\alpha m} v^{m}}{(v, v)} * \hat{\kappa}_{v}
$$


It is important that elements $\gamma_{i}$ (7.6) are central, commuting with any element of the algebra. Together with (7.5) this guarantees formal consistency of the system in the sense that (7.4) is compatible with the associativity of the star product

$$
[\mathcal{W} * \mathcal{W}, \mathcal{W}]_{*}=0
$$

and the consequence of $(7.5)$

$$
[\mathcal{W} * \mathcal{W}, \mathcal{B}]_{*}=0 .
$$

In the sector of one-forms $\mathcal{W}_{1}=W, S$ and zero-forms $\mathcal{B}_{0}=B$ this system with $F_{*}\left(\mathcal{B}, \gamma_{i}\right)$ linear in $\gamma_{i}$ reproduces (6.6)-(6.10). Higher degrees of two-forms $\gamma_{i}$ contribute to equations for the higher forms and are important for the construction of invariants as discussed below.

The construction of equations (7.4), (7.5) applies both to irreducible Coxeter systems and to the reducible ones, containing their direct products. In the latter case the label $i$ of $\gamma_{i}$ encodes both the conjugacy classes of the irreducible Coxeter systems and different factors in the direct product. This corresponds to the extension $i \rightarrow i, a$. To impose one or another symmetric reduction, $F_{*}\left(\mathcal{B}, \gamma_{i}\right)$ should be invariant under the chosen symmetry group $S$. The appearance of higher differential forms in the model is important both for its string-like interpretation since string theory is known to contain higher degree differential forms and for the construction of invariants along the lines of [136].

Invariant functionals are associated with various $x$-space forms $\mathcal{L}$ that are closed as a consequence of field equations (7.4), (7.5). As shown in [136], these can be associated with various $d Z$-independent central elements of the star-product algebra. In the case of usual HS theories considered in [136] the only relevant central element was unity of the star-product algebra. In the Coxeter HS theories of this paper the $d Z$-independent center of the algebra is generated by the elements $I_{n}^{a}$. In the simplest additive case equation (7.4) is modified to

$$
\mathcal{W} * \mathcal{W}=-i\left(d Z^{A n} d Z_{A n}+F_{*}\left(\mathcal{B}, \gamma_{i}\right)\right)+\mathcal{L}
$$

where

$$
\left.\mathcal{L}:=\sum_{n_{1}, \ldots, n_{k}, a_{1}, \ldots, a_{k}} I_{n_{1}}^{a_{1}} * \ldots * I_{n_{k}}^{a_{k}} \mathcal{L}_{a_{1}, \ldots, a_{k}}^{n_{1}, \ldots, n_{k}}(x)\right)
$$

obeys

$$
\mathrm{d} \mathcal{L}_{a_{1}, \ldots, a_{k}}^{n_{1}, \ldots, n_{k}}(x)=0 .
$$

The density forms $\mathcal{L}_{a_{1}, \ldots, a_{k}}^{n_{1}, \ldots, n_{k}}(x)$ are symmetric under the exchange of pairs of indices $n_{l}, a_{l}$

$$
\mathcal{L}_{\ldots, a_{n}, \ldots, n_{m}, \ldots, n_{m}, \ldots}^{\ldots, \ldots}(x)=\mathcal{L}_{\ldots, a_{m}, \ldots, n_{m}, \ldots, n_{n}, \ldots}, \ldots(x)
$$

and contain differential forms of any positive even degree

$$
\mathcal{L}=\mathcal{L}_{2}+\mathcal{L}_{4}+\mathcal{L}_{6}+\ldots
$$

generating invariant functionals as integrals over space-time cycles of appropriate dimension

$$
S=\int_{\Sigma} \mathcal{L}
$$


Let us stress that (7.11) is a consequence of (7.9), i.e., apart from imposing field equations on dynamical fields, eq. (7.9) expresses $\mathcal{L}$ via dynamical fields themselves in such a way that $\mathcal{L}$ turns out to be closed.

System (7.9), (7.11) and (7.5) is invariant under the following gauge transformations with three types of gauge parameters $\varepsilon, \xi$ and $\chi[136]$ :

$$
\begin{aligned}
\delta \mathcal{W} & =[\mathcal{W}, \varepsilon]_{*}+\xi^{N} \frac{\partial \mathcal{F}_{*}(\mathcal{B}, \gamma)}{\partial \mathcal{B}^{N}}+\chi_{i} \frac{\partial \mathcal{L}}{\partial \mathcal{L}_{i}}, \\
\delta \mathcal{B} & =\{\mathcal{W}, \xi\}_{*}+[\mathcal{B}, \varepsilon]_{*}, \\
\delta \mathcal{L}_{i} & =\mathrm{d} \chi_{i}
\end{aligned}
$$

where $N$ is the multiindex running over all components of $\mathcal{B}$, the gauge parameters $\varepsilon$ and $\xi$ are differential forms of even and odd degrees, respectively, being otherwise arbitrary functions of $x$ and the generating elements of the star-product algebra, while $\chi_{i}$ only depend on $x$ and $d x$. By (7.17), the functional $S$ (7.14) is gauge invariant.

Generalization of this construction to the systems with non-additive contribution of the Lagrangian forms $\mathcal{L}$ is also possible and can be constructed along the lines of [136].

\section{Color extensions}

As usual in HS theory $[5,6]$, one can let all fields be valued in a matrix algebra, i.e., carry Chan-Paton-like $[137,138]$ matrix indices. Upon imposing appropriate reality conditions the respective HS models possess $\mathrm{U}(n)$ gauge symmetry in the spin-one sector. Following the construction of $[8,139]$ (see also [6]) the resulting models can be truncated to those with $O(n)$ and $\operatorname{Sp}(2 m)$ spin-one gauge groups. (For instance $O(1)$-model is the so-called minimal HS model that only contains even spins.)

The local symmetry algebras of different types of $4 d$ HS systems of [5] are $h u(n, m \mid 8)$, $h o(n, m \mid 8)$ and $h u s p(n, m \mid 8){ }^{2}$ Analogously, local symmetry algebras of different types of $3 d$ HS systems are $h u_{\alpha \beta}(n, m \mid 4), h o_{\alpha \beta}(n, m \mid 4)$ and $h u s p_{\alpha \beta}(n, m \mid 4)(\alpha, \beta=0,1, \alpha+\beta \neq 0)[8]$.

In the both cases the meaning of these notations is that the master fields are valued in appropriate $(n+m) \times(n+m)$ matrices in which bosons belong to the diagonal $n \times n$ and $m \times m$ blocks while fermions are in the two off-diagonal $n \times m$ blocks. The respective HS models possess usual supersymmetry at $n=m$.

For multi-particle HS theories one can use algebras $M(h \ldots(n, m \mid 2 l))$. Also one can add matrix indices to $M(h \ldots(n, m \mid 2 l))$ in the end which option seems to be less interesting, however.

One proceeds analogously in the rank- $p$ Coxeter HS models. The respective algebras will be called $\mathcal{C} h \ldots(n, m \mid 2 l)$ with $\mathcal{C}$ denoting a Coxeter group in question. An interesting option available in this case is to let the fields $W$ and $B$ be valued in the matrix algebra $\left(\times M a t_{N+M}\right)^{p}$, e.g., $C^{u_{1} \ldots u_{p}} v_{1 \ldots v_{p}}\left(Y^{n}\right), u_{i}, v_{i}=1 \ldots N+M$ with the idea to assign any

\footnotetext{
${ }^{2}$ These should not be confused with the $4 d$ global symmetry algebras $h u(n, m \mid 4), h o(n, m \mid 4)$ and $h u s p(n, m \mid 4)$ of [139]. The difference expresses the doubling of spinor variables $Y \rightarrow Y, Z$ in the nonlineal system compared to the $Z$-independent dynamical fields like $\omega(Y ; K \mid x)$.
} 
pair of matrix indices $u_{n}, v_{n}$ to the variable $Y^{n}$. From the $A d S / C F T$ perspective, indices $u_{i}$ with $i=1, \ldots N$ and $i=N+1, \ldots N+M$ can be associated with the boundary scalar and spinor fields, respectively. More generally, one can consider multi-indexed fields with indices $u_{i}$ and $v_{i}$ associated with rank- $p$ tensors obeying various symmetry and/or tracelessness conditions. Still, the respective $\mathcal{C} h \ldots(n, m \mid 2 l)$ Coxeter HS theories make sense though the relation of $n$ and $m$ with $N$ and $M$ is more complicated.

Usually, indices of this type are not considered in the bulk HS models. The rationale behind this is that the singlet contributions due to the field $C^{u_{1} \ldots u_{p}} v_{1 \ldots v_{p}}\left(Y^{n}\right) \sim$ $\delta_{v_{1}}^{u_{1}} \ldots \delta_{v_{p}}^{u_{p}} C\left(Y^{n}\right)$ decouple from the traceful components, being in a certain sense dominating. This happens because invariants of the color symmetry cannot source components transforming nontrivially under the color group. Careful analysis of this phenomenon may be important for understanding specificities of the $(O(N))^{p}$-models associated with boundary fields being symmetric and/or traceless with respect to color indices (as well as their unitary and symplectic analogues) in which case the respective factors $\delta_{v_{1}}^{u_{1}} \ldots \delta_{v_{p}}^{u_{p}}$ have to be appropriately symmetrized and/or projected to the traceless components within the sets of indices $u_{1} \ldots u_{p}$ and $v_{1} \ldots v_{p}$.

\section{Idempotent extension}

In this section we propose a further extension of the construction of HS equations allowing to unify brane-like systems of different space-time dimensions in the same HS model. This construction is associated with idempotents (projectors) of the star-product algebra underlying the system in question. Usually, these are Fock idempotents. The idea is illustrated by the fact that the Fock module of the $4 d$ HS algebra describes $3 d$ conformal fields [113] associated with the boundary fields in the $A d S_{4} / C F T_{3}$ correspondence [92]. Let us stress that in presence of unbroken HS symmetries, the lower-dimensional branes of this type turn out to be delocalized, i.e., reduction of space-time dimension is via a projective identification of astigmatic type not allowing to observe certain directions. However, if the HS symmetries are broken (e.g. by boundary conditions), the HS branes can become space-time localized.

\subsection{General scheme}

The general construction is as follows. Let $A$ be some associative algebra with the product $*$ and $\pi_{i} \in A$ be a set of idempotents

$$
\pi_{i} * \pi_{i}=\pi_{i}
$$

Let $A_{i}{ }^{j} \subset A$ be subspaces of $A$ spanned by the elements of the form

$$
a_{i}{ }^{j} \in A_{i}{ }^{j}: \quad a_{i}{ }^{j}=\pi_{i} * a * \pi_{j}, \quad a \in A .
$$

The composition law is of the matrix-like form which, in turn, is a particular case of this construction

$$
(a * b)_{i}{ }^{j}=\sum_{k} a_{i}{ }^{k} * b_{k}{ }^{j}
$$


Note that in this construction the idempotents $\pi_{i}$ need not be orthogonal. The only important condition is (9.1).

The resulting idempotent-extended algebra $A_{\{\pi\}}$ is associative and unital with the unit element

$$
I d_{i}{ }^{j}=\delta_{i}^{j} \pi_{i} .
$$

If the algebra $A$ was unital it can itself be realized as a particular case of the above construction with $\pi=I d$.

A particular case of this scheme with two idempotents was used in [140] for the formulation of a $d=2$ HS theory. Here we propose its slightly different application directly to the nonlinear equations of sections 6,7 .

\subsection{Realization}

In HS theory, $A$ is the algebra of functions of $d x, d Z, Z, Y, \kappa, x$ with the star product (6.23) underlying the construction of section 6 . Idempotents $\pi_{i}$ can be identified with the $Z$ independent Fock projectors of the star-product algebra. We conjecture that exact holographic correspondence can take place for the systems which correspond to consistent nonlinear equations containing both bulk and boundary fields via the idempotent construction of this section.

\subsubsection{Vector-like models}

For instance, in the $4 d$ HS theory

$$
\pi_{i}=4 I_{i} \exp y_{i \alpha} \bar{y}_{i}^{\alpha}
$$

are such idempotents, i.e.,

$$
\pi_{i} * \pi_{i}=\pi_{i}
$$

(note that here dotted and undotted indices are on equal footing). $\pi_{i}$ can indeed be interpreted as Fock idempotents since

$$
\left(y_{i \alpha}-i \bar{y}_{i \alpha}\right) * \pi_{i}=0, \quad \pi_{i} *\left(y_{i \alpha}+i \bar{y}_{i \alpha}\right)=0 .
$$

The idempotent-extended system has the same form as the systems of sections 6,7 with the replacement of the original algebra $A$ by $A_{\{\pi\}}$. The unit element of the star-product algebra is considered as one of the idempotents, say, $\pi_{0}$. Note that with this convention $\pi_{0}$ is not orthogonal to $\pi_{i}$. Luckily, as emphasized in the previous section, they do not need to be orthogonal for the applicability of the general scheme. The important consistency condition is that the full set of idempotents has to be invariant under the Coxeter group in the system and the mutual products $\pi_{i} * \pi_{j}$ as well as the elements (9.2) should be well-defined.

In the original HS system, the vacuum solution (6.13) contains $S_{0}$ linear in $Z$ that solves (6.10) because $Z_{A}$ obey Heisenberg commutation relations (2.10). In the idempotentextended version of the Coxeter HS equations, the respective vacuum solution is

$$
S_{0}=\sum_{i} \pi_{i} * d Z^{A n} Z_{A n} * \pi_{i}
$$


where the condition that all $\pi_{i}$ are $Z$-independent and hence $*$-commute with $Z_{A n}$ implies that so defined $S_{0}$ solves (7.9) with the unit element (9.4).

If HS fields carry matrix indices, the star-product idempotents $\pi_{i}^{\text {star }}$ can be accompanied by those in color indices $\pi_{i}^{\text {color }}$

$$
\pi_{i}=\pi_{i}^{\text {star }} \pi_{i}^{\text {color }}
$$

The simplest idempotent $\pi_{i}^{\text {color }}$ is given by the diagonal matrix with a single unit element on the diagonal, like $\delta_{1}^{u} \delta_{v}^{1}$. In that case the left and right modules in this sector can be associated with the space of rows and columns with respect to the matrix algebra. This leads to the vector-like fields in $A_{0}{ }^{i}$ and $A_{i}{ }^{0}$.

The physical interpretation of the proposed construction is that the fields valued in new idempotent sectors of $A_{\{\pi\}}$ live in lower space-time dimensions compared to those valued in $A$. This is so since the HS modules supported by $A_{i}{ }^{j}$-modules are smaller (i.e., have smaller Gelfand-Kirillov dimension) than those of the twisted adjoint $A$-module supporting dynamical fields in the original system. For instance, the $4 d$ twisted adjoint module describes $4 d$ massless fields of all spins while the $A_{0}{ }^{i}$-module (9.2), (9.5) describes $3 d$ conformal fields (i.e., $4 d$ singletons) [113]. This suggests that the idempotent extensions of the Coxeter HS systems are appropriate to describe lower-dimensional objects analogous to branes in String Theory.

From the $A d S / C F T$ perspective this construction makes it possible to describe in the same model both fundamental boundary fields as valued in the $A_{0}{ }^{i}$ and $A_{i}{ }^{0}$ sectors and composite current-like operators as fundamental fields in the twisted adjoint $A$-module. Let us stress again that the simplest extension of the idempotent construction to the matrix sector gives vector-like boundary fields.

The condition that the set of idempotents $\left\{\pi_{i}\right\}$ is invariant under the action of the Coxeter group $\mathcal{C}$ restricts significantly the brane (in particular, boundary) sectors. Since the Coxeter group action exchanges different arguments $Y$ and $Z$ the condition is that all idempotents $\pi_{i}^{\text {star }}$ that belong to the same orbit of $\mathcal{C}$ should be multiplied by the same color idempotent, i.e., $\pi_{i}^{\text {color }}=\pi_{i}^{\text {color }}\left(O_{\mathcal{C}}\left(\pi_{i}^{\text {star }}\right)\right)$, where $\pi_{i}^{\text {color }}\left(O_{\mathcal{C}}\left(\pi_{i}^{\text {star }}\right)\right)$ depends on the $\mathcal{C}$-orbits of $\pi_{i}^{\text {star }}$-idempotents. For instance, if there is a $\mathcal{C}$-invariant $\pi_{i}^{\text {star }}$-idempotent, it can be multiplied by any $\pi_{i}^{\text {color }}$. On the other hand, if there are many different idempotents related by the action of $\mathcal{C}$, they should all be multiplied by the same color idempotent.

The latter situation is important in the context of usual HS holography. In this case idempotents $\pi_{i}^{\text {star }}$ associated with the boundary tensor fields have to be of the form

$$
\pi_{i}^{\text {star }}=\Pi_{i} \pi^{\text {color }},
$$

where $\Pi_{i}$ is the Fock projector in the sector of $Y_{i}$ oscillators and $\pi^{\text {color }}$ has to be the same for any $\pi_{i}^{\text {star }}$. The most natural option is to choose $\pi^{\text {color }}$ as the rank-one idempotent

$$
\delta_{1}^{u_{1}} \delta_{v_{1}}^{1} \ldots \delta_{1}^{u_{p}} \delta_{v_{p}}^{1}
$$

Here all indices $u_{1} \ldots u_{p}$ and $v_{1} \ldots v_{p}$ have to take the same number of values $N$. Correspondingly, the boundary fields are scalars or spinors of the type $\varphi^{u_{1} \ldots u_{p}}(x)$ with all indices taking $N$ values. 


\subsection{2 $N=4$ SUSY}

The original and most important example of holographic correspondence is the duality of Superstring Theory with the most supersymmetric $N=4$ SYM theory [1]. Here we briefly discuss how this correspondence can be interpreted from the perspective of the proposal of this paper suggesting that string-like HS theories are associated with the Coxeter group $B_{2}$ (see also section 12). Remarkably, the case of $N=4$ supersymmetry turns out to be distinguished from this perspective as well.

Our consideration is based on the unfolded formulation of the linearized $N=4 \mathrm{SYM}$ theory proposed in $[114,141]$ where it was shown that $4 d$ conformal massless fields can be described by the fields valued in certain Fock modules. Let the Fock vacuum $\pi_{0 \overline{0}}$ be defined by the relations

$$
\begin{array}{lll}
a_{\alpha} * \pi_{0 \overline{0}}=0, & \bar{b}^{\dot{\beta}} * \pi_{0 \overline{0}}=0, & \phi_{i} * \pi_{0 \overline{0}}=0, \\
\pi_{0 \overline{0}} * \bar{a}^{\dot{\alpha}}=0, & \pi_{0 \overline{0}} * b_{\alpha}=0, & \pi_{0 \overline{0}} * \bar{\phi}^{i}=0
\end{array}
$$

with respect to the oscillators obeying the following non-zero relations

$$
\left[a_{\alpha}, b^{\beta}\right]_{*}=\delta_{\alpha}^{\beta}, \quad\left[\bar{a}_{\dot{\gamma}}, \bar{b}^{\dot{\beta}}\right]_{*}=\delta_{\dot{\gamma}}^{\dot{\beta}}, \quad\left\{\phi_{i}, \bar{\phi}^{j}\right\}_{*}=\delta_{i}{ }^{j},
$$

$i, j=1, \ldots N$. Bilinears of these oscillators form $s u(2,2 ; N)$ which therefore acts on the Fock module where $4 d$ massless fields are valued. In fact, in [141] it was shown that, to have a room for HS potentials for massless fields, one should introduce four Fock modules generated from the Fock vacua $\pi_{p \bar{p}}(p, \bar{p}=0,1)$ obeying relations analogous to (9.12) with exchanged roles of the creation and annihilation operators $a_{\alpha}, b_{\beta}$ and $\bar{a}^{\dot{\alpha}}, \bar{b}^{\dot{\beta}}$. For instance,

$$
\begin{array}{lll}
b_{\alpha} * \pi_{1 \overline{1}}=0, & \bar{a}^{\dot{\beta}} * \pi_{1 \overline{1}}=0, & \bar{\phi}^{i} * \pi_{1 \overline{1}}=0, \\
\pi_{1 \overline{1}} * \bar{b}^{\dot{\alpha}}=0, & \pi_{1 \overline{1}} * a_{\alpha}=0, & \pi_{1 \overline{1}} * \phi_{i}=0 .
\end{array}
$$

The Clifford oscillators can be interpreted as generating the color matrix algebra $M a t_{2^{2 N}}$ where all fields are valued. Let us now explain how this construction appears in the framework of the $B_{2}$-HS model and why the case of $N=4$ is distinguished.

The $B_{2}$-HS theory contains a pair of oscillators $y_{i \alpha}, \bar{y}_{i \dot{\alpha}}$. This allows us to build oscillators analogous to $(9.7)$

$$
a_{\alpha}=y_{1 \alpha}+i y_{2 \alpha}, \quad b_{\alpha}=\frac{1}{4 i}\left(y_{1 \alpha}-i y_{2 \alpha}\right), \quad \bar{a}_{\dot{\alpha}}=\bar{y}_{1 \dot{\alpha}}-i \bar{y}_{2 \dot{\alpha}}, \quad \bar{b}_{\dot{\alpha}}=\frac{1}{4 i}\left(\bar{y}_{1 \dot{\alpha}}+i \bar{y}_{2 \dot{\alpha}}\right)
$$

defining vacuum $\pi_{00}$ (9.12). More precisely, since the commutation relations (6.3) contain $I_{n}$, to obey (9.12), (9.13) and (9.1) the idempotent $\pi_{00}$ should contain the factor of $I_{1} * I_{2}$.

The fields valued in the left and right modules generated from this vacuum describe $4 d$ massless conformal fields. To describe supermultiplets the vacuum $\pi_{0 \overline{0}}$ has to be combined with the Clifford vacuum also obeying (9.12), (9.13) [114].

Although, naively, this construction relates the $B_{2}$-HS theory to the massless conformal supermultiplets at any $N$, this is not quite the case. The problem is that the idempotent $\pi_{0 \overline{0}}$ defined this way is not invariant under the $B_{2}$ reflection exchanging $Y_{1}^{A}$ with $Y_{2}^{A}$ or 
changing a sign of one of them. Indeed, such reflections map idempotent $\pi_{0 \overline{0}}$ to the opposite idempotent $\pi_{1 \overline{1}}$. In fact, the condition that the set of idempotents has to be invariant under the action of the Coxeter group $B_{2}$ demands all $\pi_{p \bar{p}}$ be present in the model.

The problem however is that opposite idempotents may have ill-defined mutual star product so that elements $\pi_{0 \overline{0}} * a * \pi_{1 \overline{1}}$ (9.2) are ill defined. Namely, for purely bosonic opposite idempotents of this type, i.e., at $N=0, \pi_{0 \overline{0}} * \pi_{1 \overline{1}}$ is divergent. On the other hand, in the purely Clifford case the product of opposite Fock idempotents is zero. In the supersymmetric case the bosonic and fermionic contributions to the vanishing determinants in the respective Gaussian integrals resulting from the star product of the idempotents have opposite signs. The full compensation of the bosonic divergency occurs just at $N=4$ when the numbers of bosonic and fermionic oscillators are equal. (Note that for a similar reason, at $N=4$ the conformal SUSY is $p s u(2,2 ; 4)$.)

Thus the consistent rank-two conformal boundary system in the $B_{2}$-HS theory must have $N \geq 4$. The modules generated from $\pi_{p \bar{p}}$ describe $4 d$ conformal massless fields (supermultiplets) of all spins [114, 141]. It is natural to conjecture that all of them except for those in the lowest-spin $N=4 \mathrm{SYM}$ supermultiplet will become massive upon spontaneous breakdown of HS symmetries in the multi-particle $B_{2}$-HS theory. Note that simultaneously the non-zero string tension should result from the spontaneous breaking of HS symmetries

At the condition that the resulting theory is free from massless fields of spins $s \geq 2$, the only remaining option is the $N=4 \mathrm{SYM}$ multiplet. This is the case of $N=4 \mathrm{SYM}$ which is the only $N=4$ massless conformal system with spins $s \leq 1$. Thus, a spontaneously broken $B_{2}$-HS theory with non-zero string tension has a chance to be dual to $N=4 \mathrm{SYM}$.

To have a nontrivial gauge group for the $N=4$ SYM boundary theory of the spontaneously broken HS theory one has to introduce color indices additional to the Clifford algebra. This can be achieved either directly by letting all fields be valued in the matrix algebra (and its further orthogonal/symplectic reductions) or via the construction used for the vector models introducing the fields $C^{u_{1} u_{2}} v_{1} v_{2}(Y)$ and using the additional color idempotent

$$
\pi^{c o l}=\delta^{u_{1} u_{2}} \delta_{v_{1} v_{2}} \delta_{v_{1}}^{u_{1}}
$$

\section{Unitarity}

One of the advantages of the unfolded formulation of dynamical equations is that it makes symmetries manifest operating directly with modules of the symmetry $h$ underlying the model in question. In particular, linearized equations on the zero-forms in the system have a form of covariant constancy conditions

$$
D_{0} C^{I}(x)=0
$$

with $C^{I}(x)$ valued in some $h$-module $V$, and $D_{0}$ being a flat covariant derivative of $h$. Though $V$ is not unitary in the Lorentz-covariant frame, the condition that the system admits consistent quantization compatible with unitarity demands $V$ be complex equivalent to some unitary $h$-module $U$ [114]. This makes it straightforward to analyze the pattern of 
one or another unfolded system in terms of space-time symmetry algebra modules including the issue of unitarity.

Naively, the proposed Coxeter HS systems contain non-unitary sectors associated with the tensor products of the unitary HS modules with the so-called topological modules of the original HS theory, that describe non-unitary finite-dimensional modules of the AdS algebra.

For instance, in the $4 d B_{2}$-HS theory, consider a rank-two field $C\left(Y_{1}, k_{1} ; Y_{2}, k_{2}\right):=$ $C_{0,1}\left(Y_{1}, Y_{2}\right) k_{2}$ proportional to the Klein operator $k_{2}$ of the second type and independent of $k_{1}$. The covariant derivative with respect to AdS background connection contains commutator with the frame field $h(Y)$ with respect to the first argument and anticommutator with respect to the second. This is manifested in the form of covariant derivative

$$
\begin{aligned}
D_{0}\left(C_{1,2}\left(Y_{1}, Y_{2}\right)\right)= & D^{\mathrm{L}} C_{1,2}\left(Y_{1}, Y_{2}\right) \\
& -h^{\alpha \dot{\beta}}\left(y_{1 \alpha} \frac{\partial}{\partial \bar{y}_{1}^{\dot{\beta}}}+\frac{\partial}{\partial y_{1}^{\alpha}} \bar{y}_{1 \dot{\beta}}-i y_{2 \alpha} \bar{y}_{2 \dot{\beta}}+i \frac{\partial^{2}}{\partial y_{2}^{\alpha} \partial \bar{y}_{2}^{\dot{\beta}}}\right) C_{1,2}\left(Y_{1}, Y_{2}\right)
\end{aligned}
$$

with the Lorentz covariant derivative

$$
D^{\mathrm{L}} A=\mathrm{d}-\sum_{i=1,2}\left(\omega^{\alpha \beta} y_{i \alpha} \frac{\partial}{\partial y_{i}^{\beta}}+\bar{\omega}^{\dot{\alpha} \dot{\beta}} \bar{y}_{i \dot{\alpha}} \frac{\partial}{\partial \bar{y}_{i}^{\dot{\beta}}}\right) .
$$

The first term in (10.2) acts on homogeneous polynomials of $Y_{1}$ of any definite degree while the second mixes polynomials of different degrees of $Y_{2}$. This structure is tantamount to the fact that $C_{1,2}\left(Y_{1}, Y_{2}\right)$ is valued in the tensor product of the adjoint module of the HS algebra with respect to $Y_{1}$ and twisted adjoint module with respect to $Y_{2}$. The twisted adjoint module and its tensor products correspond to unitary particle-like states [142] and their multi-particle tensor products, respectively.

Having a form of the infinite sum of finite-dimensional modules, the zero-form fields valued in the adjoint module or even containing it as a factor as in (10.2) cannot correspond to a unitary particle-like representation. The only exception is when the field $C_{1,2}\left(Y_{1}, Y_{2}\right)$ is independent of $Y_{1}$. Then it contains the factor of $I_{1}$ and, in accordance with the discussion of section 6 , corresponds to unitary massless states of the usual HS theory in the sector of variables $Y_{2}$.

Naively, non-singlet states in the adjoint module factors break down unitarity of the system. However, these potentially non-unitary states can be consistently truncated away. Indeed, consider for instance product of the factors $C_{1,2}\left(Y_{1}, Y_{2}\right) k_{2}$. There are two options. Either the arguments $Y_{1}, k_{1}$ and $Y_{2}, k_{2}$ are permuted, in which case one ends up with the unitary rank-two field, or they are not permuted. In the latter case, the product will still contain the singlet component $I_{1}$ in the adjoint factor describing nonlinear corrections to usual massless HS equations, i.e., non-singlet elements of the adjoint factor are never generated. Note that, to account properly the contribution of the Klein operators, their explicit appearance in eq. (7.4) via the factors of $\gamma_{i}$ should be taken into account.

In the original HS theory topological fields were interpreted as moduli (coupling constants) rather than propagating degrees of freedom. Similarly, in the Coxeter HS theories, 
the fields associated with non-unitary sectors resulting from the tensor products of unitary and topological modules should be interpreted as non-propagating by imposing appropriate boundary conditions in the topological sector somewhat analogously to the analysis of conformal gravity in [143]. On the other hand assigning non-zero VEVs to the fields in potentially non-unitary sectors may significantly affect the interpretation of the bulk HS theory similarly to the $3 d$ HS model of [8] in which the value of the singlet topological field is interpreted as the mass parameter in the theory. Analogous suggestion for the massgenerating spontaneous breaking mechanism in multi-particle HS theories is discussed in section 12. The question whether the non-unitary fluctuations can be consistently truncated away in presence of non-zero VEVs of topological fields remains to be investigated.

A related comment is that the issue of whether a HS model is unitary or not may depend on the choice of reality conditions and vacuum solution. For instance, in the sector of zero-forms $C$ of the $4 d$ HS theory, condition (6.19) just implies that, from the perspective of the $3 d$ model, the field $C$ is valued in the tensor product of (complexified) $3 d$ particle module with the topological one. Nonetheless, the resulting module of the $4 d$ HS model turns out to be unitary due to imposing appropriate reality conditions compatible with the $4 d$ vacuum solution.

To summarize, to make the proposed models unitary, potentially dangerous fields containing products with non-singlet factors of the adjoint HS module have to be truncated away. Remarkably, this is possible in all orders so that the reduced model turns out to be unitary. This factorization is somewhat analogous to that of non-singlet color sectors in the HS theory with colorful fields.

Let us stress that the HS models dual to the free tensor boundary theories with $O\left(N^{p}\right)$ broken to $O(N)^{3}$ at the level of observables have to be unitary [78]. This condition has little to do with potential non-unitarity of interacting tensor models with ill-defined potentials. On the other hand, it would be interesting to see whether the non-unitary boundary tensor models can be dual to $B_{p}$-HS theories with non-unitary bulk fields switched on.

\section{Field frames of multi-particle theories}

In this section we recall the construction of [94] for the multi-particle algebra extending it to the graded-symmetric case and analysis of idempotents.

\subsection{Definition and field equations}

Let $A$ be an associative algebra with the product law $*$ and basis elements $t_{i}$ obeying

$$
t_{I} * t_{J}=f_{I J}^{K} t_{K}, \quad f_{I J}^{K} f_{K L}^{N}=f_{I K}^{N} f_{J L}^{K}
$$

In the Coxeter HS case, $A$ is (matrix-valued extension of) the algebra of functions of $I_{i}, Z_{i}^{A}, Y_{i}^{A}, K_{v}, d Z_{i}^{A}$ as well as of $\pi_{i}$ for the idempotent extension of section 9 (space-time differential $d x^{\underline{n}}$ is not included). 
As a linear space, multi-particle algebra $M(A)$ is the direct sum of all graded-symmetric tensor degrees of $A$

$$
M(A)=\sum_{n=0}^{\infty} \oplus S y m \underbrace{A \otimes \ldots \otimes A}_{n},
$$

where the $\mathbb{Z}_{2}$-grading is identified with the power in $Z_{i}^{A}$ and $Y_{i}^{A}$,

$$
f\left(-Z_{i}^{A},-Y_{i}^{A}\right)=(-1)^{\pi_{f}} f\left(Z_{i}^{A}, Y_{i}^{A}\right),
$$

i.e., odd polynomials in $Z_{i}^{A}$ and $Y_{i}^{A}$ are antisymmetrized under the symbol $S y m$ in (11.2). Let us stress that this does not contradict to the fact that $Z_{i}^{A}$ and $Y_{i}^{A}$ obey star-product commutation relations in $A$. So defined graded symmetrization accounting the total number of spinorial indices carried by $Z_{i}^{A}$ and $Y_{i}^{A}$ expresses the Pauli principle. (Note that in the vectorial model of [91] considered in section 6.3 only integer-spin states contribute.)

Let $\alpha_{I}$ be odd (even) elements of the Grassmann algebra associated with the odd (even) elements $t_{I}$, respectively,

$$
\alpha_{I} \cdot \alpha_{J}=(-1)^{\pi_{I} \pi_{J}} \alpha_{J} \cdot \alpha_{I} .
$$

The product · denotes usual (juxtaposition) product in the Grassmann algebra of $\alpha$ introduced to distinguish between the graded symmetric tensor product $\cdot$ and the commutative product in the definition of the star-product algebra underlying $A$ of $M(A)$. The latter is the algebra of functions $F .(\alpha)$ with the product law

$$
F .(\alpha) \circ G .(\alpha)=F .(\alpha) \cdot \exp .\left(\frac{\overleftarrow{\partial}}{\partial \alpha_{I}} f_{I J}^{N} \alpha_{N} \frac{\vec{\partial}}{\partial \alpha_{J}}\right) \cdot G .(\alpha)
$$

An elementary computation shows that associativity of $A$ implies associativity of the product $\circ$ of $M(A)$. (For the even case see [94].)

Algebra $M(A)$ is unital, with the unit element $I d$ identified with $F(\alpha)=1$. The $\mathbb{Z}_{2}$-grading of $M(A)$ is induced by that of $A$

$$
F\left((-1)^{\pi(\alpha)} \alpha\right)=(-1)^{\pi(F)} F(\alpha) .
$$

The graded commutativity (11.4) implies the graded symmetrization in (11.2).

The power-series expansion of the functions $F .(\alpha)$

$$
F .(\alpha)=\sum_{n=0}^{\infty} F^{I_{1} \ldots I_{n}} \cdot \alpha_{I_{1}} \cdot \ldots \cdot \alpha_{I_{n}}
$$

will be assumed to have anticommuting coefficients $F^{I_{1} \ldots I_{n}}$

$$
F^{I_{1} \ldots I_{n}} \cdot G^{J_{1} \ldots J_{m}}=(-1)^{\left(\sum_{k=1}^{n} \pi_{I_{k}}\right)\left(\sum_{l=1}^{m} \pi_{J_{l}}\right)} G^{J_{1} \ldots J_{m}} \cdot F^{I_{1} \ldots I_{n}} .
$$

The same time it is convenient to demand them to commute with $\alpha_{I}$

$$
F^{I_{1} \ldots I_{n}} \cdot \alpha_{J}=\alpha_{J} \cdot F^{I_{1} \ldots I_{n}} .
$$


Since components of $F .(\alpha)$ are identified with the physical fields in the multi-particle HS theory, convention (11.8) expresses the Pauli principle.

Since $A \subset M(A)$ is represented by linear functions of $\alpha_{I}$, the $*$ product acts on linear functions of $\alpha_{I}$ according to

$$
\alpha_{J} * \alpha_{K}=f_{J K}^{M} \alpha_{M} .
$$

Note that $A \circ A$ does not belong to $A$.

$M(A)$ is isomorphic to the universal enveloping algebra $\mathrm{U}(l(A))$,

$$
M(A) \sim \mathrm{U}(l(A)),
$$

where $l(A)$ is the Lie superalgebra associated with $A$,

$$
\left[t_{I}, t_{J}\right]_{*}=g_{I J}^{K} t_{K}, \quad g_{I J}^{K}=f_{I J}^{K}-(-1)^{\pi_{I} \pi_{J}} f_{J I}^{K} .
$$

This is because $M(A)$ is isomorphic to $\mathrm{U}(l(A))$ as a linear space and

$$
\alpha_{I} \circ \alpha_{J}-(-1)^{\pi_{I} \pi_{J}} \alpha_{J} \circ \alpha_{I}=g_{I J}^{K} \alpha_{K} .
$$

Concise form of the product law (11.5) is specific for the case of Lie superalgebras $l=l(A)$ associated with associative algebras $A$.

\subsection{Frame generating functions}

The following useful property of $M(A)$ is a simple consequence of eq. (11.5)

$$
\forall f, g \in A: \quad \text { exp. } f(\alpha) \circ \exp . g(\alpha)=\exp .(f \bullet g)(\alpha),
$$

where

$$
f \bullet g:=f+g+f * g=\left(f+e_{*}\right) *\left(g+e_{*}\right)-e_{*} \in A
$$

and $e_{*}$ is the unit element of $A$ if the latter is unital (recall that $f, g \in A$ implies that $f(\alpha)$ and $g(\alpha)$ are linear in $\alpha$ ). Associativity of $*$ implies associativity of $\bullet$ even if $A$ is not unital.

Let

$$
G_{\cdot \nu}=\exp .(\nu) \in M(A), \quad \nu=\nu^{I} \cdot \alpha_{I},
$$

where $\nu^{I}$ are free Grassmann-odd (even) parameters for odd (even) $\alpha_{I}$, which however commute with $\alpha_{i}$

$$
\nu^{I} \cdot \nu^{J}=(-1)^{\pi_{I} \pi_{j}} \nu^{J} \cdot \nu^{I}, \quad \nu^{I} \cdot \alpha_{J}=\alpha_{J} \cdot \nu^{I}
$$

Eq. (11.14) gives

$$
G_{\cdot \nu} \circ G_{\cdot \mu}=G_{\cdot \nu \bullet \mu} .
$$

This formula is convenient for practical computations via differentiation over $\nu^{I}$ with $G_{\cdot \nu}$ serving as the generating function for elements of $M(A)$.

As any universal enveloping algebra $M(A)$ is double filtered. Indeed, let $V_{n}$ be the linear space of order- $n$ polynomials of $\alpha_{I}$. From eqs. (11.5), (11.13) it follows that

$$
F_{n} \circ F_{m} \in V_{n+m}, \quad F_{n} \circ F_{m}-(-1)^{\pi\left(F_{n}\right) \pi\left(F_{m}\right)} F_{m} \circ F_{n} \in V_{n+m-1}, \quad F_{m} \in V_{m}, \quad F_{n} \in V_{n} .
$$


A linear map of $M(A)$ to itself can be represented by

$$
\varkappa(\alpha, a)[F .]=\varkappa\left(\alpha, \frac{\vec{\partial}}{\partial \alpha}\right) F .(\alpha)
$$

where derivatives $\frac{\vec{\partial}}{\partial \alpha}$ act on $F .(\alpha)$. This formula can be interpreted as representing the action of the normal-ordered oscillator algebra with the generating elements $\alpha_{I}$ and $\bar{\alpha}^{J}$ acting on the Fock module spanned by $F(\alpha)|0\rangle$ with $\bar{\alpha}^{I}|0\rangle=0$. To respect the double filtration property mapping order- $n$ polynomials to order- $n$ polynomials, $\varkappa(\alpha, \bar{\alpha})$ should be filtered obeying

$\varkappa(\alpha, \bar{\alpha})=\sum_{m, n=0}^{\infty} \varkappa_{I_{1} \ldots I_{m}}{ }_{J_{1} \ldots J_{n}} \alpha_{I_{1}} \cdot \ldots \cdot \alpha_{I_{m}} \cdot \bar{\alpha}^{J_{1}} \cdot \ldots \cdot \bar{\alpha}^{J_{n}}, \varkappa^{I_{1} \ldots I_{m}} J_{1} \ldots J_{n}=0$ at $m>n$.

In these terms, the unit map is $\mathbf{I} \mathbf{d}=1$. Consider maps of the form

$$
U(f) \equiv \varkappa(\alpha, \bar{\alpha} \mid f)=\phi \exp .\left(\alpha_{I} \cdot f^{I}(\bar{\alpha})\right)
$$

with some $\alpha$-independent coefficients $f_{\text {. }}^{I}(\bar{\alpha})$ and constant $\phi$. The map $U(f$.) is filtered if $f^{I}(\bar{\alpha})$ is at least linear in $\bar{\alpha}$, i.e.,

$$
f^{I}(0)=0 .
$$

Interpreting $\bar{\alpha}$ as parameters, we can identify any $f(\alpha):=\sum_{I} f^{I} \alpha_{I}$ with $f(t)=$ $\sum_{I} f^{I} t_{I} \in A$. For $U(f)$ (11.22) acting on $G_{\cdot \nu}(11.16)$ we obtain

$$
U(f)\left(G_{\cdot \nu}\right)=\exp .\left(\tilde{f}^{I}(\nu) \alpha_{I}\right), \quad \tilde{f}^{I}(\nu)=\nu^{I}+f^{I}(\nu) .
$$

Hence, eq. (11.14) gives

$$
U(f)\left(G_{\cdot \nu}\right) \circ U(g)(G \cdot \mu)=\exp .((\tilde{f}(\nu) \bullet \tilde{g}(\mu))(\alpha)),
$$

where $\tilde{f}(\nu)$ and $\tilde{g}(\mu)$ are interpreted as elements of $A$, i.e., $\tilde{f}(\nu)=\tilde{f}^{I}(\nu) \alpha_{I}$.

Consider map (11.22) with

$$
f^{I}(\bar{\alpha}) \alpha_{I}=f\left(a_{n}\right), \quad a_{n}:=\alpha_{I_{1}} * \ldots * \alpha_{I_{n}} \cdot \bar{\alpha}^{I_{1}} \ldots . \bar{\alpha}^{I_{n}}, \quad a_{1}=a=\alpha_{I} \cdot \bar{\alpha}^{I}, \quad a_{0}=e_{*},
$$

where $f\left(a_{n}\right)$ is a linear function of $a_{n}(n \geq 1)$. Such a map has the form (11.22) since $a_{n} \in A$. A particularly important class of maps (11.22) is represented by $\mathbf{U}_{u}$ of the form

$$
\mathbf{U}_{u}(a)=: \exp [u(a)-a]:,
$$

where normal ordering is with respect to $\alpha_{I}$ and $\bar{\alpha}^{I}$ and for $a \in A$

$$
u(a)=\left(u_{1}^{1} a+u_{1}^{2} e_{*}\right) *\left(u_{2}^{1} a+u_{2}^{2} e_{*}\right)_{*}^{-1}, \quad\left(e_{*}+\beta a\right)_{*}^{-1}:=\sum_{n=0}^{\infty}(-\beta)^{n} a_{n}
$$

with $u_{i}^{j} \in \mathbb{R}$. Composition of such maps gives a map of the same class

$$
\mathbf{U}_{u} \mathbf{U}_{v}=\mathbf{U}_{u v}
$$


where $(u v)_{i}^{j}=u_{i}{ }^{k} v_{k}{ }^{j}$ is the matrix product. The maps $\mathbf{U}_{u}$ with $\operatorname{det}|u| \neq 0$ are invertible and form Mobius group.

From eq. (11.24) it follows that

$$
\mathbf{U}_{u}\left(G_{\cdot \nu}\right)=G_{\cdot u(\nu)} \cdot
$$

To find the composition law of $M(A)$ in the frame $T_{I_{1} \ldots I_{n}}^{u}$ associated with $G_{\cdot u(\nu)}$ via

$$
T_{I_{1} \ldots I_{n}}^{u}=\left.\frac{\partial^{n}}{\partial \nu^{I_{1}} \ldots \partial \nu^{I_{n}}} G_{\cdot u(\nu)}\right|_{\nu=0}
$$

we compute

$$
G_{\cdot \nu} \diamond G_{\cdot \mu}:=\mathbf{U}_{u}^{-1}\left(G_{\cdot u(\nu)} \circ G_{\cdot u(\mu)}\right) .
$$

eq. (11.15) gives

$$
G_{\cdot \nu} \diamond G_{\cdot \mu}=G_{\cdot u^{-1}(u(\nu) \bullet u(\mu))} \cdot
$$

General map (11.27) is not filtered, not respecting condition (11.23). The subgroup $P$ of filtered maps (11.27) is represented by the lower triangular matrices

$$
u_{b, \beta}(f)=b f *\left(e_{*}+\beta f\right)_{*}^{-1}
$$

with the composition law

$$
b_{1,2}=b_{1} b_{2}, \quad \beta_{1,2}=\beta_{2}+\beta_{1} b_{2} .
$$

Using for these transformations notation $U_{b, \beta}$ instead of $U_{u}$ we observe that the unit element is

$$
\mathbf{I d}=\mathbf{U}_{1,0}
$$

and

$$
\mathbf{U}_{b, \beta}^{-1}=\mathbf{U}_{b^{-1},-\beta b^{-1}}
$$

The map

$$
\mathbf{R}=\mathbf{U}_{-1,1}
$$

is involutive

$$
\mathbf{R}^{2}=\mathbf{I d}
$$

describing the principal antiautomorphism of $M(A)$ [94].

For the maps (11.34), the composition law (11.33) takes the form [94]

$$
G_{\cdot \nu} \diamond G_{\cdot \mu}=G_{\cdot \sigma_{b, \beta}(\nu, \mu)},
$$

where

$$
\sigma_{b, \beta}(\nu, \mu)=-\beta^{-1}\left(e_{*}-\left(e_{*}+\beta \mu\right) *\left(e_{*}-\beta(b+\beta) \nu * \mu\right)^{-1} *\left(e_{*}+\beta \nu\right)\right) .
$$

Three most important cases include

$$
\begin{aligned}
\sigma_{1,0}(\nu, \mu) & =\nu+\mu+\nu * \mu=\nu \bullet \mu \\
\sigma_{-1,1}(\nu, \mu) & =\nu+\mu+\mu * \nu=\mu \bullet \nu, \\
\sigma_{1,-\frac{1}{2}}(\nu, \mu) & =2\left(e_{*}-\left(2 e_{*}-\mu\right) *\left(4 e_{*}+\nu * \mu\right)_{*}^{-1} *\left(2 e_{*}-\nu\right)\right) .
\end{aligned}
$$


Here $\sigma_{1,0}(\nu, \mu)$ corresponds to the identity map reproducing the basis in $M(A)$ resulting just from the symmetrized tensor product of the framed oscillator algebras. $\sigma_{-1,1}(\nu, \mu)$ corresponds to the basis resulting from the action of principal antiautomorphism $\mathbf{R}$. The case of $\sigma_{1,-\frac{1}{2}}(\nu, \mu)$ is most interesting, reproducing the current operator algebra of [144].

Practically, the difference between the frames (11.42) and (11.44) is as follows. The product law associated with (11.42) is the original product law (11.4), from which it is easy to see that the product $\circ$ of two polynomials of $\alpha$ of degrees $n$ and $m$ gives degree- $k$ polynomials with $\max (n, m) \leq k \leq n+m$. This implies that, with the product law (11.4), the higher-rank fields (say, rank-two) will not contribute to the equations for the lower-rank ones (say, rank-one). This property is inconsistent both with structure of the boundary operator algebra analyzed in [144] and with the idea that VEVs of the higher-rank fields can modify field equation for the lower-rank ones to yield a Higgs phenomenon. The product law associated with (11.43) has analogous properties.

However, in [94] it was shown that the multi-particle algebra in the frame (11.44) properly reproduces the boundary operator algebra inducing such a product law that the product of two polynomials of $\alpha$ of degrees $n$ and $m$ gives degree- $k$ polynomials with $|m-n| \leq k \leq n+m$ which is consistent both with the structure of the boundary operator algebra of [144] and with the idea of higgsing by virtue of VEVs of higher-rank fields. Hence, we anticipate that frame (11.44) is most appropriate for the analysis of the multiparticle Coxeter HS theories. Let us stress that since the multi-particle algebra is infinite dimensional it is not a priori guaranteed that the multi-particle HS theories associated with different frames described in this section are physically equivalent.

In application to nonlinear equations of the multi-particle HS theory, system (7.4), (7.5) preserves its form with the $*$ replaced by $\diamond$

$$
\mathcal{W} \diamond \mathcal{W}=-i\left(d Z^{A n} d Z_{A n}+F_{\diamond}\left(\mathcal{B}, \gamma_{i}\right)\right), \quad[\mathcal{W}, \mathcal{B}]_{\diamond}=0
$$

where $\diamond$ is built via (11.44) from the star product of the Coxeter HS theory underlying its multi-particle extension.

\subsection{Unity idempotent map}

Multi-particle algebra has an important property that every idempotent $\pi \in A$ induces an idempotent $\Pi \in M(A)$. This immediately follows from formulae (11.14), (11.15) with

$$
\Pi:=\exp .-\pi
$$

i.e., $\Pi \circ \Pi=\Pi$ if $\pi * \pi=\pi$. In particular, application of this construction to $\pi=e$ gives the unity idempotent of $M(A)$

$$
\Pi_{e}:=\exp .-e .
$$

Note that being built from the central element $e, \Pi_{e}$ is central in $M(A)$.

Unity idempotent $\Pi_{e}$ has an interesting property that it is $\circ$-orthogonal to any $a \in A$

$$
\Pi_{e} \circ a=a \circ \Pi_{e}=0, \quad a \in A,
$$


which is obvious from (11.5) and (11.47). Analogously one can check that

$$
\left(a \cdot \Pi_{e}\right) \circ b=(a * b) \cdot \Pi_{e}, \quad \forall a \in A
$$

and

$$
a \cdot \Pi_{e} \circ b \cdot \Pi_{e}=a * b \cdot \Pi_{e}, \quad \forall a, b \in A .
$$

This relation provides a homomorphism of $A$ to $M(A)$. It should be stressed however that this map is not polynomial. (No homomorphism of $A$ to $M(A)$ realized as polynomial functions of $A$ exists.)

Relation (11.50) has the consequence that

$$
\Pi_{e}^{1}:=e \cdot \Pi_{e}
$$

is also an idempotent

$$
\Pi_{e}^{1} \circ \Pi_{e}^{1}=\Pi_{e}^{1}
$$

From (11.49) it follows

$$
\Pi_{e}^{1} \circ a=a \cdot \Pi_{e}, \quad \forall a \in A .
$$

The consequence of this construction is that if $A$ had a Lie (super)algebra $l$ associated with some $t_{i} \in A$, then $M(A)$ admits a symmetry algebra $l \oplus l$ generated by $t_{i}$ and $t_{i} \cdot \Pi_{e}$ with $t_{i}$ generating the diagonal subalgebra $l \subset l \oplus l$. This fact may have important implications for the space-time interpretation of the string-like HS theories discussed in the next section.

\section{Interpretation}

\subsection{Strings and tensor models}

Coxeter HS equations and their multi-particle extensions have a number of features indicating their relation to string-like models and their further tensor-like extensions anticipated to be holographic duals of the boundary tensor sigma-models considered in [77, 78].

First of all, the spectra of fields described by the rank- $p$ Coxeter HS models with $p>1$ are far larger than of usual rank-one HS gauge theories. This is most obvious from the fact that the zero-form fields $C\left(Y_{\alpha}^{n} ; k_{v}\right)$ depend on $p$ copies of the oscillators $Y_{\alpha}^{n}$ as well as on the Klein operators $k_{v}$ associated with all roots of the underlying Coxeter system (modulo identification $k_{-v}=k_{v}$ ). This enlargement of the spectrum is in qualitative agreement with the observation of $[78,81]$ that the spectrum of the boundary operators in tensor boundary models is far richer than in the vector sigma-model. Most importantly, however, the Klein operators generating Coxeter reflections effectively permute the arguments of the elementary master fields like $C\left(Y_{1}, \ldots Y_{p} ; k \mid x\right)$.

In the absence of the Klein operators, products of the fields would correspond to the tensor product of $p$ copies of star-product algebras valued in $M a t_{N+M}$. However, in the process of solving Coxeter HS equations, relations of the type (3.22) will permute the variables $Y_{n}$ and $Y_{m}$ with different $n$ and $m$, not affecting the matrix indices $a_{n}, b_{n}$ 
and $a_{m}, b_{m}$. For instance, in the case of $p=2$, the star product of two master fields $C\left(Y_{1}, Y_{2} \mid x\right) k_{12}$ gives

$$
\left(C\left(Y_{1}, Y_{2} \mid x\right) k_{12}\right) *\left(C\left(Y_{1}, Y_{2} \mid x\right) k_{12}\right)=C\left(Y_{1}, Y_{2} \mid x\right) * C\left(Y_{2}, Y_{1} \mid x\right) .
$$

As a result, nonlinear corrections to the $p=2$ system will contain products of elementary strings of master fields with repeatedly permuted arguments $Y_{1}$ and $Y_{2}$

$$
C_{\text {string }}^{n}:=\underbrace{C\left(Y_{1}, Y_{2} \mid x\right) * C\left(Y_{2}, Y_{1} \mid x\right) * C\left(Y_{1}, Y_{2} \mid x\right) \ldots}_{n} .
$$

Such strings are analogous to the product of elementary matrix factors and can be identified with the letters of an infinite Alphabet with $n=0,1, \ldots \infty$. These are analogues of the single-trace operators in the ordinary $A d S / C F T$ dictionary. General product of operators decomposes into products of elementary letters analogous to the multi-trace operators. For instance, operator (12.1) as well as $C\left(Y_{1}, Y_{2} \mid x\right)$ are single-trace while $C\left(Y_{1}, Y_{2} \mid x\right) * C\left(Y_{1}, Y_{2} \mid x\right)$ is double-trace. Thus the spectrum of operators of the $p=2$ HS model is analogous to that of String Theory with the infinite set of Regge trajectories. More precisely, to interpret these nonlinear combinations of operators as corresponding to elementary states of some string-like model one has to consider a multi-particle HS model associated with the infinite set of elementary $B_{2}$ systems

$$
\operatorname{sym}\left(B_{2} \times B_{2} \times \ldots\right),
$$

where the graded symmetrization is with respect to all elementary $B_{2}$ factors in the sense that all respective master fields $C\left(Y_{1}^{1}, Y_{2}^{1}, k_{v}^{1} ; Y_{1}^{2}, Y_{2}^{2}, k_{v}^{2} ; Y_{1}^{3}, Y_{2}^{3}, k_{v}^{3} ; \ldots \mid x\right)$ are demanded to be graded symmetric under the exchange of the variables $Y_{1}^{a}, Y_{2}^{a}, k_{v}^{a}$ associated with different factors of $B_{2}$ (i.e., index $a$ ).

The $p=2$ Coxeter HS theory has deep parallels with the analysis of stringy HS models by Gaberdiel and Gopakumar [70]-[74]. In particular, the master fields of this theory depend on the two sets of oscillators $Y_{1,2}^{A}$ which is close to saying that the stringy HS theory is based on two different HS symmetry algebras being one of the conclusions of Gaberdiel and Gopakumar. It should be stressed that these algebras do not commute with each other if the stringy coupling constant identified with the vacuum expectation value of $F_{2 *}(B)$ in (6.16) is non-zero inducing nontrivial Cherednik-like deformation (5.10) of the oscillator commutation relations. (Note that for conformal models considered by Gaberdiel and Gopakumar the VEV of $F_{1 *}(B)$ must be non-zero.)

Usual HS theory and its multi-particle extension result from the analogous construction applied to $B_{1}$. Rank- $p$ tensor HS theories and their multi-particle extensions result analogously from $B_{p}$ with $p>2$ as well as from other higher-rank Coxeter root systems. Clearly the pattern of elementary operators of the $p>2$ higher-rank Coxeter HS models will increase enormously compared to the $p=2$ string-like models which is in agreement with $[78,81]$.

To make the holographic correspondence more explicit it is necessary to compare the spectrum of singlet boundary operators with that of the singlet sector of the tensor bulk 
model. As explained in section 6, due to using framed algebras, these spectra do match at least in the massless sector containing usual massless states dual to the boundary currents in the holographic interpretation. The pattern of the full spectrum of the higher-rank Coxeter HS theories remains to be elaborated.

Let us stress that the Coxeter HS theories proposed in this paper are formulated in the anti-de Sitter space of appropriate dimension. In particular, this is true for the stringy $B_{2}$-HS models. Hence the construction of this paper is different from that of genuine String Theory formulated in the flat rather than, say, $A d S_{10}$ space. In fact, the reason why it is difficult to formulate String Theory in $A d S_{d}$ is analogous to that discussed in section 4 for HS theory: a naive attempt to deform the string spectrum to AdS would immediately lead to infinite vacuum energy since in this case all string modes have to contribute to the momentum generators to ensure that their commutator is proportional to the Lorentz ones

$$
\left[P_{n}, P_{m}\right] \sim-\Lambda M_{n m}
$$

that act on all modes. (Recall that the usual string theory momentum operator is built from the zero modes [19] which is not possible in AdS.) Hence, the extension to the framed oscillator algebra can also be crucial to reach a string-like theory in AdS.

An important related feature of the $B_{p}$-HS models with $p \geq 2$, is that they have two independent coupling constants instead of one in the usual $B_{1}$-HS theories. These are the coefficients $\eta_{1,2}$ in the linearized parts of the functions $F_{1,2 *}(B)$ in (6.12). The function $F_{1 *}$ is analogous to that of the rank-one (i.e., $B_{1}$ ) HS theory. The respective term is important for the proper interpretation of the equations in $A d S_{4}$ leading to the so-called central on-shell theorem of [142] representing the unfolded equations for massless fields in $A d S_{4}$. The function $F_{2 *}$ first appears in the rank-two stringy model and, containing the Klein operators that permute different species of $Y$-variables, is responsible for the appearance of single-trace-like strings of operators. The presence of two different coupling constants is anticipated to have important implications for establishing relation with usual string theory in flat space. One option is that to reach the latter theory one has to take the limit with $\eta_{2} / \eta_{1} \rightarrow \infty$ which in turn may select String Theory in critical dimension.

It should be stressed that it is a distinguishing property of the $B_{p} \sim C_{p}$ Coxeter group that the related Cherednik system has two types of coupling constants responsible for HS and stringy effects. It is not clear, in particular, whether the $A_{p}$ and $D_{p}$ systems admit a meaningful HS interpretation since, due to the absence of usual Klein operators reflecting signs of different species of oscillators $Y_{A}^{i}$, the free field equations unlikely have a room for massless fields.

The full-fledged string theory is conjectured to be related to a rank-two multi-particle $B_{2}$ Coxeter HS model. As explained in section 11 following [94], the form of the multiparticle algebra significantly depends on the chosen basis. It remains to be analysed to which extent the formulations in different frames are equivalent to each other. More precisely, different frames of the Coxeter HS models of finite rank are equivalent. However, for the multi-particle extensions the respective frame changes are in the infinite-dimensional space and different frames may not be equivalent. The details of description of multi- 
particle algebras in different frames are presented in section 11 where the construction of [94] is extended to the graded-symmetric case expressing the Pauli principle.

This question may be related to the fundamental issue of the breaking of HS symmetries in the Coxeter HS theory. Indeed, it is plausible to expect $[145,146]$ that spontaneous breaking of HS symmetries resulting in the appearance of massive HS fields is only possible in string-like models with the infinite number of Regge trajectories. As conjectured in this paper the appropriate Coxeter HS theory is the $B_{2}$ multi-particle theory. The goal is to break down HS symmetries to usual space-time symmetry of AdS or Minkowski type. The simplest way to do so is to let a rank-two zero-form topological field $B\left(Y_{1} ; Y_{2}\right)$ acquire a non-zero VEV

$$
B_{0}=Y_{i A} \cdot Y_{j}^{A}\left(\gamma \sigma_{1}^{i j}+\rho \delta^{i j}\right),
$$

where the Pauli matrix $\sigma_{1}^{i j}$ and $\delta^{i j}$ are the two symmetric matrices invariant under the exchange $1 \leftrightarrow 2$ in $i, j$. (Note that this proposal is somewhat reminiscent of the GirardelloPorrati-Zaffaroni mechanism [147].) Such $B_{0}$ preserves the AdS symmetry but breaks down the HS one. Note that so defined $B_{0}$ is nonzero because elementary oscillators $Y^{i A}$ are anticommuting with respect to the product - (11.4). It remains to be seen how this VEV would affect (deform) the structure of the AdS modules (field equations) of the originally massless fields. Spontaneous symmetry breaking would correspond to the mixture between the originally massless rank-one particle module and the rank-two current module. In the infinite-dimensional case of the multi-particle algebra the mechanism of such mixing may depend on the choice of the frame in the multi-particle algebra. As argued in section 11.2, it is natural to anticipate that the proper frame is defined by (11.44), being associated with the boundary current algebra of [144]. Detailed analysis of this issue is one of the most urgent problems on the agenda.

\section{$12.2 \quad$ Space-time metamorphoses}

The formulation of HS equations in the unfolded form expressing the space-time exterior derivative $\mathrm{d}$ via the values of other fields like in equations (2.3)-(2.5) on the fields $W, B$ and $S$ subjected to the constraints (2.6) and (2.7) allows us to unify in the same framework the systems that live in space-times of different dimensions [92, 124, 128]. This is achieved by letting the de Rham derivative $\mathrm{d}$ be defined in the infinite-dimensional space-time. Then the physical space-times like Minkowski or AdS appear when the background (vacuum) connection $W_{0}(x)$ of the respective symmetry group $G$ is nontrivial with non-degenerate frame-like components along $x_{\|}^{n}$ associated with the translation (or transvection) generators. In that case the coordinates $x_{\|}^{n}$ are observable while the rest ones $x_{\perp}^{\frac{n}{\perp}}$ are not: in the absence of components of forms $W_{0}(x)$ along $x_{\perp}^{\underline{n}}$ the respective unfolded equations treated perturbatively would imply that, locally, all other differential form fields in the system are either $x_{\perp}^{n}$-independent zero-forms or pure gauge (i.e., exact) $p>0$-forms. By this mechanism, the system formulated in the infinite-dimensional space-time is visualized by the coordinates $x_{\|}^{n}$ representing one or another $G$-invariant space described by $W_{0}\left(x_{\|}^{n}\right)$.

In [124] it was observed that the higher-rank fields in lower dimension can be interpreted as elementary fields in higher dimension. In the framework of Coxeter HS theory 
this phenomenon acquires a direct realization as we explain now. Consider a spinorial Coxeter HS theory. In this case, the rank-one sector consists of the fields depending on a single spinor variable $Y^{A}$ with $A=1, \ldots M(M=2$ in the $3 d$ model and $M=4$ in the $4 d$ model). Bilinears of $Y^{A}$ form generators of $s p(M)$ with respect to the star product. This is the $A d S_{4}$ algebra $s p(4)$ in the $4 d$ model and a $s p(2)$ half of the $A d S_{3}$ algebra in the $3 d$ model (to be doubled via introducing the Clifford element $\psi_{1}[8]$ ). The diagonal embedding of the star-product generators of $s p^{\operatorname{diag}}(M)$ into a rank- $p$ system is

$$
t_{A B}=\sum_{i=1}^{p}\left\{Y_{A i}, Y_{B i}\right\}_{*} .
$$

Vacuum connection $W_{0}(x)$ that describes usual space-time geometry like $A d S_{4}$ or $A d S_{3}$ is a flat $s p(M)$ connection $(s p(2) \oplus s p(2)$ in the $3 d$ case).

Let $\Omega=A, i$. Operators

$$
T_{\Omega \Lambda}=\left\{Y_{\Omega}, Y_{\Lambda}\right\}_{*} * I_{1} * \ldots * I_{p}
$$

are generators of $s p(p M)$. The diagonal embedding $s p^{\operatorname{diag}}(M)$ into $s p(p M)$ is realized by the generators

$$
t_{A B}^{\prime}=t_{A B} * I_{1} * \ldots * I_{p} .
$$

The same time the $s p(M)$ generated by $t_{A B}$ (12.6) acts diagonally on the full framed Cherednik algebra. Evidently,

$$
\left(t_{A B}-t_{A B}^{\prime}\right) * T_{\Omega \Lambda}=T_{\Omega \Lambda} *\left(t_{A B}-t_{A B}^{\prime}\right)=0 .
$$

Let $\tau_{\Omega^{\prime} \Lambda^{\prime}}$ form a basis of $s p(p M) / s p^{\operatorname{diag}}(M)$. Due to (12.9), it is possible to identify the space-time components of the flat connections of $t_{A B}$ and $t_{A B}^{\prime}$ demanding them to have nonzero components along coordinates $x \frac{n}{\|}$ of the space-time $\mathcal{M}$ originally associated with the $s p(M)$ symmetry. Now it is possible to choose a flat connection $W_{0}^{\prime}(X)$ on some $s p^{\prime}(p M)$ invariant space-time $\mathcal{M}^{\prime}$ with local coordinates $X$ in such a way that the pushforward of $W_{0}^{\prime}(X)$ to $\mathcal{M}$ gives $W_{0}(x)$. This makes it possible to treat the rank- $p$ fields in $\mathcal{M}$ as elementary fields in $\mathcal{M}^{\prime}$.

One can proceed analogously with the additional species of oscillators in the multiparticle algebra construction. From the analysis of section 11.3 it follows that the multiparticle $B_{2}$-HS model has $s p(8) \oplus s p(8)$ as a finite-dimensional symmetry. The natural homogeneous space that admits this symmetry is the group manifold $\operatorname{Sp}(8)$ which is 36dimensional. Moreover, the conformal-like symmetry of $\operatorname{Sp}(8)$ is $\operatorname{Sp}(16)[114,148,149]$.

This has an interesting consequence that the usual space-time interpretation of the Coxeter HS theories and their multi-particle extensions is likely to have maximal Minkowski dimension ten. This follows from the analysis of the $s p(2 M)$ invariant theories in $[126,127]$ where it was shown how usual Minkowski space-time dimensions emerges from the $s p(2 M)$ invariant equations with the conclusion that the maximal Minkowski space known to emerge from the $s p(16)$-invariant equations is ten dimensional. Though it is tempting to conjecture that this is how the Superstring dimension ten emerges from the Coxeter HS theory, details 
of this phenomenon need further investigation. In particular, it would be interesting to see whether this phenomenon is related to the twistor-like transform in ten dimensions introduced by Witten in [130].

Note that the mechanism explained in this section is based on the extension of the space-time symmetries due to appearance of the additional species of oscillators in the higher-rank Coxeter HS theories and/or multi-particle extension of HS theories. As such it is not quite the same as the holographic correspondence within the idempotent construction of section 9 where the symmetry algebra $g$ remains the same but the $g$-module pattern changes depending on the idempotent sector in question.

\section{Conclusion}

We propose a class of Coxeter HS models conjectured to underly a symmetric phase of String Theory (Coxeter group $B_{2}$ ) and its further tensor-like extensions (Coxeter group $B_{p}$ ). These models contain two coupling constants one of which is responsible for stringy effects (absent in the conventional HS theory) and exhibit a number of interesting parallelisms with String Theory. In particular, consistency of the holographic interpretation of the boundary matrix-like model demands the latter to have $N=4$ SUSY.

The main idea of our construction was to find a formally consistent extension of the known HS gauge systems [5, 8, 91] possessing a richer spectrum and having a room for massless fields of all spins including spin-two gravitational field. The former goal was reached via extension of the Coxeter groups $Z_{2}$ or $Z_{2} \times Z_{2}$ generated by the Klein elements of the models of $[5,8,91]$ to any Coxeter group including the most important case of $B_{p}$. To let massless fields be present in the model this construction was further extended to the framed algebras containing additional idempotent elements.

Though the main emphasize in this paper was on the spinorial HS models somewhat analogous to Green-Schwarz superstring, bosonic Coxeter HS theories of vectorial type considered in section 6.3 and their fermionic counterparts to be elaborated are also of interest as analogues of the bosonic and fermionic strings. (For more detail see [134].) We believe that formal consistency in presence massless fields in the spectrum is so restrictive that it hardly leaves a room for consistent HS models beyond the list presented in this paper, supplemented by the Coxeter extensions of to be constructed fermionic generalizations of the model of [91] in any dimension. In particular it determines the structure of extended HS symmetries as well as the field pattern.

Many of the important aspects of the proposed models, such as detailed analysis of field spectra, spontaneous breaking of HS symmetries, holographic interpretation, proper space-time interpretation, the choice of appropriate frame in the multi-particle HS theories and others were only briefly sketched in this paper, demanding a more detailed study delegated to the future as well as some other issues including, for instance, the analysis of locality along the lines of $[17,18]$.

An interesting open question is to give an interpretation to the Coxeter HS theories based on the Coxeter groups different from $B_{p}$ which were of most interest in this paper. In particular, it would be interesting to study more carefully the case of Dihedral group 
$I_{2}(n)$ which is the symmetry group of the $n$-gone on the plane. In this relation it should be noted that the case of $B_{2}$ is special due to the isomorphism $B_{2} \sim I_{2}(4)$. An interesting feature of the $B_{2} \sim I_{2}(4)$ HS theory is that, as shown recently in [150, 151], for certain linear relations between the coupling constants $\nu_{1}$ and $\nu_{2}$ the respective Cherednik algebra acquires ideals. As a result, on this locus of the plane of coupling constants some states in the system should decouple. It would be interesting to investigate this phenomenon in detail, especially in the context of unitarity.

\section{Acknowledgments}

I am grateful to Nima Arkani-Hamed, Matthias Gaberdiel, Olga Gelfond, Igor Klebanov, Arkady Tseytlin, Herman Verlinde and especially Semyon Konstein for useful discussions. This research was supported by the Russian Science Foundation Grant No 18-12-00507.

Open Access. This article is distributed under the terms of the Creative Commons Attribution License (CC-BY 4.0), which permits any use, distribution and reproduction in any medium, provided the original author(s) and source are credited.

\section{References}

[1] J.M. Maldacena, The large $N$ limit of superconformal field theories and supergravity, Int. J. Theor. Phys. 38 (1999) 1113 [Int. J. Theor. Phys. 38 (1999) 1113] [hep-th/9711200] [INSPIRE].

[2] S.S. Gubser, I.R. Klebanov and A.M. Polyakov, Gauge theory correlators from noncritical string theory, Phys. Lett. B 428 (1998) 105 [hep-th/9802109] [INSPIRE].

[3] E. Witten, Anti-de Sitter space and holography, Adv. Theor. Math. Phys. 2 (1998) 253 [hep-th/9802150] [INSPIRE].

[4] M.A. Vasiliev, Consistent equation for interacting gauge fields of all spins in (3+1)-dimensions, Phys. Lett. B 243 (1990) 378 [inSPIRE].

[5] M.A. Vasiliev, More on equations of motion for interacting massless fields of all spins in (3+1)-dimensions, Phys. Lett. B 285 (1992) 225 [INSPIRE].

[6] M.A. Vasiliev, Higher spin gauge theories: star product and AdS space, hep-th/9910096 [INSPIRE].

[7] E.S. Fradkin and M.A. Vasiliev, On the gravitational interaction of massless higher spin fields, Phys. Lett. B 189 (1987) 89 [INSPIRE].

[8] S.F. Prokushkin and M.A. Vasiliev, Higher spin gauge interactions for massive matter fields in 3D AdS space-time, Nucl. Phys. B 545 (1999) 385 [hep-th/9806236] [INSPIRE].

[9] S.F. Prokushkin and M.A. Vasiliev, Cohomology of arbitrary spin currents in AdS $S_{3}$, Theor. Math. Phys. 123 (2000) 415 [hep-th/9907020] [INSPIRE].

[10] M.A. Vasiliev, Star-product functions in higher-spin theory and locality, JHEP 06 (2015) 031 [arXiv: 1502.02271] [INSPIRE].

[11] N. Boulanger, P. Kessel, E.D. Skvortsov and M. Taronna, Higher spin interactions in four-dimensions: Vasiliev versus Fronsdal, J. Phys. A 49 (2016) 095402 [arXiv: 1508.04139] [INSPIRE]. 
[12] X. Bekaert, J. Erdmenger, D. Ponomarev and C. Sleight, Quartic AdS interactions in higher-spin gravity from conformal field theory, JHEP 11 (2015) 149 [arXiv:1508.04292] [INSPIRE].

[13] E.D. Skvortsov and M. Taronna, On locality, holography and unfolding, JHEP 11 (2015) 044 [arXiv: 1508.04764] [INSPIRE].

[14] M.A. Vasiliev, Current interactions and holography from the 0-form sector of nonlinear higher-spin equations, JHEP 10 (2017) 111 [arXiv:1605.02662] [INSPIRE].

[15] M.A. Vasiliev, On the local frame in nonlinear higher-spin equations, JHEP 01 (2018) 062 [arXiv: 1707.03735] [INSPIRE].

[16] D. Ponomarev, A note on (non)-locality in holographic higher spin theories, Universe 4 (2018) 2 [arXiv: 1710.00403] [INSPIRE].

[17] O.A. Gelfond and M.A. Vasiliev, Homotopy operators and locality theorems in higher-spin equations, arXiv:1805.11941 [INSPIRE].

[18] V.E. Didenko, O.A. Gelfond, A.V. Korybut and M.A. Vasiliev, Homotopy properties and lower-order vertices in higher-spin equations, arXiv:1807.00001.

[19] M.B. Green, J.H. Schwarz and E. Witten, Superstring theory. Volume 1: introduction, Cambridge University Press, Cambridge U.K. (1987).

[20] D.J. Gross and P.F. Mende, String theory beyond the Planck scale, Nucl. Phys. B 303 (1988) 407 [INSPIRE].

[21] D.J. Gross, High-Energy Symmetries of String Theory, Phys. Rev. Lett. 60 (1988) 1229 [INSPIRE].

[22] M.A. Vasiliev, Massless fields of all spins in the Anti-de Sitter space and their gravitational interaction, in the proceedings of the $21^{\text {st }}$ International Symposium on Theory of Elementary Particles, October 12-16, Sellin, Germany (1987).

[23] S.E. Konshtein and M.A. Vasiliev, Massless representations and admissibility condition for higher spin superalgebras, Nucl. Phys. B 312 (1989) 402 [InSPIRE].

[24] R.R. Metsaev, IIB supergravity and various aspects of light cone formalism in AdS space-time, hep-th/0002008 [INSPIRE].

[25] A. Sagnotti, Notes on strings and higher spins, J. Phys. A 46 (2013) 214006 [arXiv: 1112.4285] [INSPIRE].

[26] M. Bianchi, J.F. Morales and H. Samtleben, On stringy $A d S_{5} \times S^{5}$ and higher spin holography, JHEP 07 (2003) 062 [hep-th/0305052] [INSPIRE].

[27] N. Beisert, M. Bianchi, J.F. Morales and H. Samtleben, Higher spin symmetry and $N=4$ SYM, JHEP 07 (2004) 058 [hep-th/0405057] [INSPIRE].

[28] M. Bianchi, Higher spin symmetry (breaking) in $N=4$ SYM theory and holography, Comptes Rendus Physique 5 (2004) 1091 [hep-th/0409292] [INSPIRE].

[29] M. Bianchi and V. Didenko, Massive higher spin multiplets and holography, hep-th/0502220 [INSPIRE].

[30] U. Lindström and M. Zabzine, Tensionless strings, WZW models at critical level and massless higher spin fields, Phys. Lett. B 584 (2004) 178 [hep-th/0305098] [INSPIRE]. 
[31] G. Bonelli, On the tensionless limit of bosonic strings, infinite symmetries and higher spins, Nucl. Phys. B 669 (2003) 159 [hep-th/0305155] [INSPIRE].

[32] A. Sagnotti and M. Tsulaia, On higher spins and the tensionless limit of string theory, Nucl. Phys. B 682 (2004) 83 [hep-th/0311257] [INSPIRE].

[33] J. Engquist and P. Sundell, Brane partons and singleton strings, Nucl. Phys. B 752 (2006) 206 [hep-th/0508124] [INSPIRE].

[34] J. Engquist, P. Sundell and L. Tamassia, On singleton composites in non-compact WZW models, JHEP 02 (2007) 097 [hep-th/0701051] [INSPIRE].

[35] S.E. Konstein, M.A. Vasiliev and V.N. Zaikin, Conformal higher spin currents in any dimension and AdS/CFT correspondence, JHEP 12 (2000) 018 [hep-th/0010239] [INSPIRE].

[36] B. Sundborg, Stringy gravity, interacting tensionless strings and massless higher spins, Nucl. Phys. Proc. Suppl. 102 (2001) 113 [hep-th/0103247] [inSPIRE].

[37] E. Witten, Spacetime reconstruction, talk at the John Schwarz $60^{\text {th }}$ birthday symposium, November 3-4, California Institute of Technology, U.S.A. (2001).

[38] A. Mikhailov, Notes on higher spin symmetries, hep-th/0201019 [INSPIRE].

[39] E. Sezgin and P. Sundell, Massless higher spins and holography, Nucl. Phys. B 644 (2002) 303 [Erratum ibid. B 660 (2003) 403] [hep-th/0205131] [INSPIRE].

[40] M. Flato and C. Fronsdal, One massless particle equals two Dirac singletons: elementary particles in a curved space. 6., Lett. Math. Phys. 2 (1978) 421 [INSPIRE].

[41] I.R. Klebanov and A.M. Polyakov, AdS dual of the critical $\mathrm{O}(N)$ vector model, Phys. Lett. B 550 (2002) 213 [hep-th/0210114] [INSPIRE].

[42] S. Giombi and X. Yin, Higher spin gauge theory and holography: the three-point functions, JHEP 09 (2010) 115 [arXiv:0912.3462] [inSPIRE].

[43] S. Giombi and X. Yin, Higher spins in AdS and twistorial holography, JHEP 04 (2011) 086 [arXiv: 1004.3736] [INSPIRE].

[44] J. Maldacena and A. Zhiboedov, Constraining conformal field theories with a higher spin symmetry, J. Phys. A 46 (2013) 214011 [arXiv:1112.1016] [inSPIRE].

[45] J. Maldacena and A. Zhiboedov, Constraining conformal field theories with a slightly broken higher spin symmetry, Class. Quant. Grav. 30 (2013) 104003 [arXiv:1204.3882] [InSPIRE].

[46] O. Aharony, G. Gur-Ari and R. Yacoby, $d=3$ Bosonic Vector Models Coupled to Chern-Simons Gauge Theories, JHEP 03 (2012) 037 [arXiv:1110.4382] [INSPIRE].

[47] R.G. Leigh and A.C. Petkou, Holography of the $N=1$ higher spin theory on AdS $S_{4}$, JHEP 06 (2003) 011 [hep-th/0304217] [INSPIRE].

[48] E. Sezgin and P. Sundell, Holography in $4 D$ (super) higher spin theories and a test via cubic scalar couplings, JHEP 07 (2005) 044 [hep-th/0305040] [INSPIRE].

[49] S. Giombi et al., Chern-Simons theory with vector fermion matter, Eur. Phys. J. C 72 (2012) 2112 [arXiv: 1110.4386] [INSPIRE].

[50] S. Giombi and X. Yin, The higher spin/vector model duality, J. Phys. A 46 (2013) 214003 [arXiv:1208.4036] [INSPIRE]. 
[51] E. Sezgin, E.D. Skvortsov and Y. Zhu, Chern-Simons matter theories and higher spin gravity, JHEP 07 (2017) 133 [arXiv:1705. 03197] [INSPIRE].

[52] V.E. Didenko and M.A. Vasiliev, Test of the local form of higher-spin equations via AdS/CFT, Phys. Lett. B 775 (2017) 352 [arXiv:1705.03440] [INSPIRE].

[53] S. Jain, S. Minwalla and S. Yokoyama, Chern Simons duality with a fundamental boson and fermion, JHEP 11 (2013) 037 [arXiv:1305.7235] [INSPIRE].

[54] G. Gur-Ari and R. Yacoby, Three dimensional bosonization from supersymmetry, JHEP 11 (2015) 013 [arXiv: 1507.04378] [INSPIRE].

[55] N. Seiberg, T. Senthil, C. Wang and E. Witten, A duality web in $2+1$ dimensions and condensed matter physics, Annals Phys. 374 (2016) 395 [arXiv:1606.01989] [INSPIRE].

[56] N. Misuna, On current contribution to Fronsdal equations, Phys. Lett. B 778 (2018) 71 [arXiv: 1706. 04605] [INSPIRE].

[57] O.A. Gelfond and M.A. Vasiliev, Current interactions from the one-form sector of nonlinear higher-spin equations, Nucl. Phys. B 931 (2018) 383 [arXiv:1706.03718] [INSPIRE].

[58] M.R. Gaberdiel and R. Gopakumar, An AdS 3 dual for minimal model CFTs, Phys. Rev. D 83 (2011) 066007 [arXiv:1011.2986] [InSPIRE].

[59] M.R. Gaberdiel and R. Gopakumar, Minimal Model Holography, J. Phys. A 46 (2013) 214002 [arXiv: 1207.6697 ] [INSPIRE].

[60] M. Henneaux and S.-J. Rey, Nonlinear $W_{\infty}$ as asymptotic symmetry of three-dimensional higher spin Anti-de Sitter gravity, JHEP 12 (2010) 007 [arXiv:1008.4579] [INSPIRE].

[61] A. Campoleoni, S. Fredenhagen, S. Pfenninger and S. Theisen, Asymptotic symmetries of three-dimensional gravity coupled to higher-spin fields, JHEP 11 (2010) 007 [arXiv: 1008.4744] [INSPIRE].

[62] M.R. Gaberdiel and T. Hartman, Symmetries of holographic minimal models, JHEP 05 (2011) 031 [arXiv:1101.2910] [InSPIRE].

[63] C. Ahn, The large $N$ 't Hooft limit of coset minimal models, JHEP 10 (2011) 125 [arXiv:1106.0351] [INSPIRE].

[64] M.R. Gaberdiel, R. Gopakumar, T. Hartman and S. Raju, Partition functions of holographic minimal models, JHEP 08 (2011) 077 [arXiv:1106.1897] [INSPIRE].

[65] C.-M. Chang and X. Yin, Higher spin gravity with matter in $A d S_{3}$ and its CFT dual, JHEP 10 (2012) 024 [arXiv: 1106.2580] [INSPIRE].

[66] A. Campoleoni, S. Fredenhagen and S. Pfenninger, Asymptotic W-symmetries in three-dimensional higher-spin gauge theories, JHEP 09 (2011) 113 [arXiv:1107.0290] [INSPIRE].

[67] P. Kraus and E. Perlmutter, Partition functions of higher spin black holes and their CFT duals, JHEP 11 (2011) 061 [arXiv:1108.2567] [INSPIRE].

[68] M. Ammon, P. Kraus and E. Perlmutter, Scalar fields and three-point functions in D = 3 higher spin gravity, JHEP 07 (2012) 113 [arXiv:1111.3926] [INSPIRE].

[69] M. Beccaria, C. Candu, M.R. Gaberdiel and M. Groher, $N=1$ extension of minimal model holography, JHEP 07 (2013) 174 [arXiv:1305.1048] [INSPIRE]. 
[70] M.R. Gaberdiel and R. Gopakumar, Higher spins \& strings, JHEP 11 (2014) 044 [arXiv:1406.6103] [INSPIRE].

[71] M.R. Gaberdiel and R. Gopakumar, Stringy symmetries and the higher spin square, J. Phys. A 48 (2015) 185402 [arXiv:1501.07236] [INSPIRE].

[72] M.R. Gaberdiel, C. Peng and I.G. Zadeh, Higgsing the stringy higher spin symmetry, JHEP 10 (2015) 101 [arXiv: 1506.02045] [INSPIRE].

[73] M.R. Gaberdiel and R. Gopakumar, String theory as a higher spin theory, JHEP 09 (2016) 085 [arXiv: 1512.07237] [INSPIRE].

[74] M.R. Gaberdiel, R. Gopakumar, W. Li and C. Peng, Higher spins and Yangian symmetries, JHEP 04 (2017) 152 [arXiv:1702.05100] [INSPIRE].

[75] M.R. Gaberdiel and R. Gopakumar, Tensionless string spectra on $A d S_{3}, J H E P 05$ (2018) 085 [arXiv: 1803.04423] [INSPIRE].

[76] G. Giribet et al., Superstrings on AdS3 at $k=1$, arXiv:1803.04420 [INSPIRE].

[77] I.R. Klebanov and G. Tarnopolsky, Uncolored random tensors, melon diagrams and the Sachdev-Ye-Kitaev models, Phys. Rev. D 95 (2017) 046004 [arXiv:1611.08915] [InSPIRE].

[78] M. Beccaria and A.A. Tseytlin, Partition function of free conformal fields in 3-plet representation, JHEP 05 (2017) 053 [arXiv: 1703.04460] [INSPIRE].

[79] I.R. Klebanov and G. Tarnopolsky, On large $N$ limit of symmetric traceless tensor models, JHEP 10 (2017) 037 [arXiv:1706.00839] [INSPIRE].

[80] S. Giombi, I.R. Klebanov and G. Tarnopolsky, Bosonic tensor models at large $N$ and small

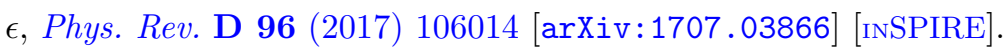

[81] K. Bulycheva, I.R. Klebanov, A. Milekhin and G. Tarnopolsky, Spectra of operators in large $N$ tensor models, Phys. Rev. D 97 (2018) 026016 [arXiv:1707.09347] [InSPIRE].

[82] E. Witten, An SYK-like model without disorder, arXiv:1610.09758 [INSPIRE].

[83] S. Sachdev and J. Ye, Gapless spin fluid ground state in a random, quantum Heisenberg magnet, Phys. Rev. Lett. 70 (1993) 3339 [cond-mat/9212030] [INSPIRE].

[84] A. Kitaev, A simple model of quantum holography, talks given at KITP, April 7 and May 27 (2015).

[85] J. Maldacena and D. Stanford, Remarks on the Sachdev-Ye-Kitaev model, Phys. Rev. D 94 (2016) 106002 [arXiv: 1604.07818] [INSPIRE].

[86] D.J. Gross and V. Rosenhaus, A generalization of Sachdev-Ye-Kitaev, JHEP 02 (2017) 093 [arXiv: 1610.01569] [INSPIRE].

[87] D.J. Gross and V. Rosenhaus, The bulk dual of SYK: cubic couplings, JHEP 05 (2017) 092 [arXiv:1702.08016] [INSPIRE].

[88] D.J. Gross and V. Rosenhaus, A line of CFTs: from generalized free fields to SYK, JHEP 07 (2017) 086 [arXiv: 1706.07015] [INSPIRE].

[89] D.J. Gross and V. Rosenhaus, All point correlation functions in SYK, JHEP 12 (2017) 148 [arXiv: 1710.08113] [INSPIRE].

[90] R. de Mello Koch, R. Mello Koch, D. Gossman and L. Tribelhorn, Gauge invariants, correlators and holography in bosonic and fermionic tensor models, JHEP 09 (2017) 011 [arXiv:1707.01455] [INSPIRE]. 
[91] M.A. Vasiliev, Nonlinear equations for symmetric massless higher spin fields in (A)dS(d), Phys. Lett. B 567 (2003) 139 [hep-th/0304049] [INSPIRE].

[92] M.A. Vasiliev, Holography, unfolding and higher-spin theory, J. Phys. A 46 (2013) 214013 [arXiv: 1203.5554] [INSPIRE].

[93] S. Giombi et al., AdS description of induced higher-spin gauge theory, JHEP 10 (2013) 016 [arXiv:1306.5242] [INSPIRE].

[94] M.A. Vasiliev, Multiparticle extension of the higher-spin algebra, Class. Quant. Grav. 30 (2013) 104006 [arXiv:1212.6071] [INSPIRE].

[95] A.P. Polychronakos, Exchange operator formalism for integrable systems of particles, Phys. Rev. Lett. 69 (1992) 703 [hep-th/9202057] [INSPIRE].

[96] L. Brink, T.H. Hansson and M.A. Vasiliev, Explicit solution to the $N$ body Calogero problem, Phys. Lett. B 286 (1992) 109 [hep-th/9206049] [INSPIRE].

[97] L. Brink, T.H. Hansson, S. Konstein and M.A. Vasiliev, The Calogero model: anyonic representation, fermionic extension and supersymmetry, Nucl. Phys. B 401 (1993) 591 [hep-th/9302023] [INSPIRE].

[98] I. Cherednik, Double affine Hecke algebras, London Mathematical Society Lecture Note Series 319, Cambridge University Press, Cambridge U.K. (2005).

[99] N. Bourbaki, Elements of mathematics, Lie groups and Lie algebras. Chapters 4-6, Springer, Germany (2002).

[100] M.A. Vasiliev, Higher spin algebras and quantization on the sphere and hyperboloid, Int. J. Mod. Phys. A 6 (1991) 1115 [inSPIRE].

[101] E. Wigner, Do the equations of motion define the quantum mechanical commutation relations?, Phys. Rev. D 77 (1950) 711.

[102] L.M. Yang, A note on the quantum rule of the harmonic oscillator, Phys. Rev. D 84 (1951) 788.

[103] S. Deser and D.G. Boulware, Ambiguity of harmonic oscillator commutation relations, Nuovo Cim. 30 (1963) 23.

[104] N. Mukunda, E.C.G. Sudarshan, J.K. Sharma and C.L. Mehta, Representations and properties of parabose oscillator operators. I. Energy position and momentum eigenstates, J. Math. Phys. 21 (1980) 2386 [INSPIRE].

[105] R.R. Metsaev, Cubic interaction vertices of totally symmetric and mixed symmetry massless representations of the Poincaré group in D =6 space-time, Phys. Lett. B 309 (1993) 39 [INSPIRE].

[106] R.R. Metsaev, Cubic interaction vertices of massive and massless higher spin fields, Nucl. Phys. B 759 (2006) 147 [hep-th/0512342] [INSPIRE].

[107] R.R. Metsaev, Cubic interaction vertices for fermionic and bosonic arbitrary spin fields, Nucl. Phys. B 859 (2012) 13 [arXiv:0712.3526] [INSPIRE].

[108] K. Alkalaev, FV-type action for AdS $S_{5}$ mixed-symmetry fields, JHEP 03 (2011) 031 [arXiv: 1011.6109] [INSPIRE].

[109] N. Boulanger, E.D. Skvortsov and Yu.M. Zinoviev, Gravitational cubic interactions for a simple mixed-symmetry gauge field in AdS and flat backgrounds, J. Phys. A 44 (2011) 415403 [arXiv: 1107.1872] [INSPIRE]. 
[110] Yu. M. Zinoviev, Gravitational cubic interactions for a massive mixed symmetry gauge field, Class. Quant. Grav. 29 (2012) 015013 [arXiv: 1107.3222] [INSPIRE].

[111] R.R. Metsaev, BRST-BV approach to cubic interaction vertices for massive and massless higher-spin fields, Phys. Lett. B 720 (2013) 237 [arXiv:1205.3131] [INSPIRE].

[112] M. Günaydin, Singleton and doubleton supermultiplets of space-time supergroups and infinite spin superalgebras, in the proceedings of the Trieste Conference "Supermembranes and Physics in 2+1 Dimensions", July 17-21, Trieste, Italy (1989).

[113] O.V. Shaynkman and M.A. Vasiliev, Higher spin conformal symmetry for matter fields in (2+1)-dimensions, Theor. Math. Phys. 128 (2001) 1155 [Teor. Mat. Fiz. 128 (2001) 378] [hep-th/0103208] [INSPIRE].

[114] M.A. Vasiliev, Conformal higher spin symmetries of $4 D$ massless supermultiplets and $\operatorname{OSp}(L, 2 M)$ invariant equations in generalized (super)space, Phys. Rev. D 66 (2002) 066006 [hep-th/0106149] [INSPIRE].

[115] P.A.M. Dirac, A remarkable representation of the $3+2$ de Sitter group, J. Math. Phys. 4 (1963) 901 [INSPIRE].

[116] C. Fronsdal, Singletons and massless, integral spin fields on de Sitter space, Phys. Rev. D 20 (1979) 848 [INSPIRE].

[117] M. Günaydin and N.P. Warner, Unitary supermultiplets of $\mathrm{OSp}(8 / 4, r)$ and the spectrum of the $S_{7}$ compactification of eleven-dimensional supergravity, Nucl. Phys. B 272 (1986) 99 [INSPIRE].

[118] E. Bergshoeff, A. Salam, E. Sezgin and Y. Tanii, Singletons, higher spin massless states and the supermembrane, Phys. Lett. B 205 (1988) 237 [INSPIRE].

[119] S. Ferrara and C. Fronsdal, Gauge fields as composite boundary excitations, Phys. Lett. B 433 (1998) 19 [hep-th/9802126] [INSPIRE].

[120] I. Bars and M. Günaydin, Unitary representations of noncompact supergroups, Commun. Math. Phys. 91 (1983) 31 [INSPIRE].

[121] A. Achucarro and P.K. Townsend, A Chern-Simons action for three-dimensional Anti-de Sitter supergravity theories, Phys. Lett. B 180 (1986) 89 [InSPIRE].

[122] E. Witten, (2+1)-dimensional gravity as an exactly soluble system, Nucl. Phys. B 311 (1988) 46 [INSPIRE].

[123] O.A. Gelfond and M.A. Vasiliev, Higher rank conformal fields in the $\operatorname{Sp}(2 M)$ symmetric generalized space-time, Theor. Math. Phys. 145 (2005) 1400 [Teor. Mat. Fiz. 145 (2005) 35] [hep-th/0304020] [INSPIRE].

[124] O.A. Gelfond and M.A. Vasiliev, Unfolded equations for current interactions of $4 D$ massless fields as a free system in mixed dimensions, J. Exp. Theor. Phys. 120 (2015) 484 [arXiv: 1012.3143] [INSPIRE].

[125] I.A. Bandos, J. Lukierski and D.P. Sorokin, Superparticle models with tensorial central charges, Phys. Rev. D 61 (2000) 045002 [hep-th/9904109] [INSPIRE].

[126] M.A. Vasiliev, Relativity, causality, locality, quantization and duality in the $S(p)(2 M)$ invariant generalized space-time, in Multiple facets of quantization and supersymmetry, M. Olshanetsky et al., World Scientific, Singapore (2002), hep-th/0111119 [INSPIRE]. 
[127] I. Bandos et al., Dynamics of higher spin fields and tensorial space, JHEP 05 (2005) 031 [hep-th/0501113] [INSPIRE].

[128] M.A. Vasiliev, Higher-spin theory and space-time metamorphoses, Lect. Notes Phys. 892 (2015) 227 [arXiv: 1404.1948] [INSPIRE].

[129] D. Sorokin and M. Tsulaia, Higher spin fields in hyperspace. A review, Universe 4 (2018) 7 [arXiv: 1710.08244$]$ [INSPIRE].

[130] E. Witten, Twistor-like transform in ten-dimensions, Nucl. Phys. B 266 (1986) 245 [INSPIRE].

[131] M.P. Blencowe, A consistent interacting massless higher spin field theory in $D=(2+1)$, Class. Quant. Grav. 6 (1989) 443 [INSPIRE].

[132] V.E. Lopatin and M.A. Vasiliev, Free Massless Bosonic Fields of Arbitrary Spin in d-dimensional de Sitter Space, Mod. Phys. Lett. A 3 (1988) 257 [inSPIRE].

[133] M.A. Vasiliev, Cubic interactions of bosonic higher spin gauge fields in AdS $S_{5}$, Nucl. Phys. B 616 (2001) 106 [Erratum ibid. B 652 (2003) 407] [hep-th/0106200] [INSPIRE].

[134] M.A. Vasiliev, Higher spin superalgebras in any dimension and their representations, JHEP 12 (2004) 046 [hep-th/0404124] [INSPIRE].

[135] M. Grigoriev and E.D. Skvortsov, Type-B formal higher spin gravity, JHEP 05 (2018) 138 [arXiv: 1804.03196] [INSPIRE].

[136] M.A. Vasiliev, Invariant functionals in higher-spin theory, Nucl. Phys. B 916 (2017) 219 [arXiv: 1504. 07289] [INSPIRE].

[137] J.E. Paton and H.-M. Chan, Generalized veneziano model with isospin, Nucl. Phys. B 10 (1969) 516 [INSPIRE].

[138] N. Marcus and A. Sagnotti, Group theory from quarks at the ends of strings, Phys. Lett. B 188 (1987) 58 [INSPIRE].

[139] S.E. Konstein and M.A. Vasiliev, Extended higher spin superalgebras and their massless representations, Nucl. Phys. B 331 (1990) 475 [INSPIRE].

[140] M.A. Vasiliev, Higher spin gauge interactions for matter fields in two-dimensions, Phys. Lett. B 363 (1995) 51 [hep-th/9511063] [INSPIRE].

[141] M.A. Vasiliev, On conformal, $\mathrm{SL}(4, \mathbb{R})$ and $\mathrm{Sp}(8, R)$ symmetries of $4 d$ massless fields, Nucl. Phys. B 793 (2008) 469 [arXiv:0707.1085] [INSPIRE].

[142] M.A. Vasiliev, Consistent equations for interacting massless fields of all spins in the first order in curvatures, Annals Phys. 190 (1989) 59 [INSPIRE].

[143] J. Maldacena, Einstein gravity from conformal gravity, arXiv:1105.5632 [INSPIRE].

[144] O.A. Gelfond and M.A. Vasiliev, Operator algebra of free conformal currents via twistors, Nucl. Phys. B 876 (2013) 871 [arXiv:1301.3123] [INSPIRE].

[145] S. Caron-Huot, Z. Komargodski, A. Sever and A. Zhiboedov, Strings from massive higher spins: the asymptotic uniqueness of the Veneziano amplitude, JHEP 10 (2017) 026 [arXiv: 1607.04253] [INSPIRE].

[146] A. Sever and A. Zhiboedov, On fine structure of strings: the universal correction to the Veneziano amplitude, JHEP 06 (2018) 054 [arXiv:1707.05270] [INSPIRE]. 
[147] L. Girardello, M. Porrati and A. Zaffaroni, $3-D$ interacting CFTs and generalized Higgs phenomenon in higher spin theories on AdS, Phys. Lett. B 561 (2003) 289 [hep-th/0212181] [INSPIRE].

[148] V.E. Didenko and M.A. Vasiliev, Free field dynamics in the generalized AdS (super)space, J. Math. Phys. 45 (2004) 197 [hep-th/0301054] [INSPIRE].

[149] M. Plyushchay, D. Sorokin and M. Tsulaia, Higher spins from tensorial charges and OSp (N-2n) symmetry, JHEP 04 (2003) 013 [hep-th/0301067] [INSPIRE].

[150] S.E. Konstein and I.V. Tyutin, Ideals generated by traces or by supertraces in the algebra of symplectic reflections $H_{1, \nu_{1}, \nu_{2}}\left(I_{2}(2 k+1)\right)$, J. Nonlin. Math. Phys. 24 (2017) 3 [arXiv: 1612.00536].

[151] S.E. Konstein and I.V. Tyutin, Ideals generated by traces in the algebra of symplectic reflections $H_{1, \nu_{1}, \nu_{2}}\left(I_{2}(2 m)\right)$, Theor. Math. Phys. 187 (2016) 706. 